\title{
Fatty acids in harp seal blubber do not necessarily reflect their diet
}

\author{
Otto Grahl-Nielsen ${ }^{1, *}$, Tore Haug ${ }^{2}$, Ulf Lindstrøm ${ }^{2}$, Kjell T. Nilssen ${ }^{2}$ \\ ${ }^{1}$ Department of Chemistry, University of Bergen, 5007 Bergen, Norway \\ ${ }^{2}$ Institute of Marine Research, PO Box 6404, 9294 Tromsø, Norway
}

\begin{abstract}
The predator-prey relationship with respect to fatty acids (FAs) was studied in 57 harp seals Pagophilus groenlandicus and 16 potential prey species collected simultaneously in the northwestern Barents Sea in May-June 2006. The FA composition of potential prey and the inner and outer sections of the seal blubber was determined. The blubber was stratified with a higher abundance of monounsaturated FAs (14 to 18 carbons) in the outer layer and a higher abundance of saturated FAs and monounsaturated long-chain FAs (20 to 24 FAs) in the inner layer. The FA composition differed substantially among potential prey species and between the prey and the blubber. The FA composition of the prey species, which was identified from stomach and/or intestine contents, was more similar to that of the blubber than that of the prey not found in the stomach or intestines. The FA composition of the outer blubber layer was independent of prey composition but dependent on the age of the seals. The weak predator-prey relationship with respect to FA composition of the inner blubber and the prey suggested that the FA composition of the inner layer was mainly predetermined by metabolism rather than the FA composition of the diet. Thus, using blubber FA composition as an estimation of prey use appears unreliable.
\end{abstract}

KEY WORDS: Harp seals · Pagophilus groenlandicus · Blubber fatty acids · Blubber stratification · Predator-prey relationship

Resale or republication not permitted without written consent of the publisher

\section{INTRODUCTION}

Harp seals Pagophilus groenlandicus are, after northeast Arctic cod Gadus morhua, the most important high trophic-level predator in the Barents Sea (Bogstad et al. 2000, Nilssen et al. 2000). The species is wide-ranging, pelagic and migratory, and forms 3 stocks inhabiting the North Atlantic Ocean. Whelping occurs on the pack ice off Newfoundland and in the Gulf of St. Lawrence (Northwest Atlantic stock), off the east coast of Greenland (Greenland Sea or West Ice stock) and in the White Sea (Barents Sea or East Ice stock) (Lavigne \& Kovacs 1988, Sergeant 1991). During spring, harp seals display a set sequence of activities: whelping (March), followed by a period of intensive lactation, after $12 \mathrm{~d}$ of which the pups are weaned and then mating occurs. Moulting of adults and immature seals takes place north of each whelping location after a further lapse of $\sim 4 \mathrm{wk}$. The location of these events is in the White Sea and southeastern parts of the Barents Sea for the East Ice stock (Haug et al. 1994, Potelov et al. 2003). At the end of the moulting period (May), the seals disperse in small herds and migrate to the feeding areas in the Svalbard waters and in the northern Barents Sea (Nordøy et al. 2008). In addition, seals from the West Ice stock migrate into the Barents Sea during summer and autumn, and satellite tracking experiments have shown that adults from the West Ice and Barents Sea stocks overlap in distribution during substantial parts of the year (July-October) (Folkow et al. 2004, Nordøy et al. 2008).

Because of seasonal variation in availability and quality of food, the body mass of harp seals varies substantially on an annual basis. There is a regular seasonal pattern of deposition of energy reserves as fat in the subcutaneous blubber layer. Harp seals are generally thin in spring (May); after moulting, however, their body condition improves over the summer, and the 
seals are relatively fat by September-October. The energy stores built up during the summer and autumn are maintained until February, but then the seals become thinner as the stores of blubber decrease rapidly during the breeding and moulting period (March-May) (Nilssen et al. 1997). The Barents Sea harp seals display opportunistic feeding patterns in that different prey species are consumed in different areas and at different times of the year as a result of spatial heterogeneity in prey availability. Nonetheless, the bulk of the harp seal diet has been observed to comprise relatively few species, in particular capelin Mallotus villosus, polar cod Boreogadus saida, krill (Thysanoessa sp. and Meganyctiphanes norvegica) and the pelagic amphipod Parathemisto libellula (Nilssen et al. 2000). In May-October, when most harp seals are distributed in the central and northern parts of the Barents Sea, they use the sea-ice edge as a platform from which they conduct foraging trips into open waters where commercially important fish species such as capelin and Arctic cod are distributed (Bergstad et al. 1987, Haug et al. 1994, Gjøsæter 1998, Nordøy et al. 2008, Gjøsæter et al. 2009).

To investigate harp seal prey use during the most intensive feeding period, diet data were sampled in open and pack-ice waters in the northern parts of the Barents Sea in May-July in 1996, 1997 and 2004-2006. The present study was based on contents of gastrointestinal tracts from 195 seals and 123 faeces, and preliminary results suggested that harp seal prey use varied both in time and space during the summer. Krill was overall the most important prey, followed by polar cod, whereas other gadoids - such as Arctic cod and haddock Melanogrammus aeglefinus-and capelin were exploited less intensively (Lindstrøm et al. 2006, Pettersen 2007). All data were obtained from animals taken near the ice edge and from faeces taken on the ice. Seal digestion is rapid, and diet studies based on contents found in faeces and in the gastrointestinal tract will only give a 'snapshot' picture of the very last meal before capture (Nilssen et al. 1995, Tollit et al. 2003, Haug et al. 2004). If the seals had visited and fed on other prey items in areas more distant from the ice prior to capture, this would most likely have remained undocumented, given the diet study methods applied. So, even though previous studies had revealed scarce occurrence of harp seals in open waters in May-July (Lindstrøm et al. 2006, Pettersen 2007), an alternative method that could give information about the dietary intake over longer time periods would be a useful supplement to the traditional diet analyses. One such alternative method is to study fatty acids (FAs) in the seal blubber. Tissue FAs have been used as trophic markers based on the theory that FAs synthesized by primary and some secondary producers are transferred in unchanged proportions through food chains (Dalsgaard et al. 2003). This approach has been applied in a long range of investigations on FA composition of marine mammal blubber (Iverson et al. 2004, Tucker et al. 2009a,b) with the assumption that changes in the FA composition of the blubber are caused by changes in diet.

Four different investigations on the impact of FAs in the prey on the FA composition of harp seal blubber have been published (Kirsch et al. 2000, Tucker et al. 2009a, Falk-Petersen et al. 2004, 2009). The first of these investigations concerned captive harp seals, and the FA composition of the seal blubber was compared with that of the diet. In the other investigations, the FA composition of the blubber was compared with that of prey species caught at other times and in other areas than the seals. Attempts to identify the prey species on the basis of the FA composition of the blubber have, however, led to varied results.

Thus, the majority of the investigations on predator-prey relationships for marine mammals have been conducted on predators and potential prey sampled at different areas and times. Investigations where the known prey have been analysed together with the blubber of the predator include studies on captive animals (Grahl-Nielsen \& Mjaavatten 1991, Kirsch et al. 2000, Iverson et al. 2004, Nordstrom et al. 2008), studies of nursing pups and their milk (Grahl-Nielsen et al. 2000, Iverson et al. 2004, Birkeland et al. 2005) and studies where animals and prey were collected simultaneously in the same area (Olsen \& Grahl-Nielsen 2003, Andersen et al. 2004, Grahl-Nielsen et al. 2005, 2010). In all these studies there were clear-cut differences between the FA composition of the blubber and of the prey. This indicates that the FA profile of the blubber is species-specific, determined by the metabolism of the animals and not by their prey.

We secured blubber samples from 57 harp seals and 16 potential prey species collected simultaneously from the northern feeding grounds in the Barents Sea. By comparing the FA composition of the blubber with that of the potential prey, we aimed to test the hypothesis that the FA composition of the blubber is independent of the prey and is determined mainly by the seal's metabolism.

\section{MATERIALS AND METHODS}

Sample collection. The material originates from a dedicated research expedition on the RV 'Jan Mayen' to the northwestern Barents Sea during the period 20 May to 8 June 2006 (Fig. 1). The survey was designed to (1) sample harp seals for studies of their feeding habits and (2) estimate the abundance of potential prey 


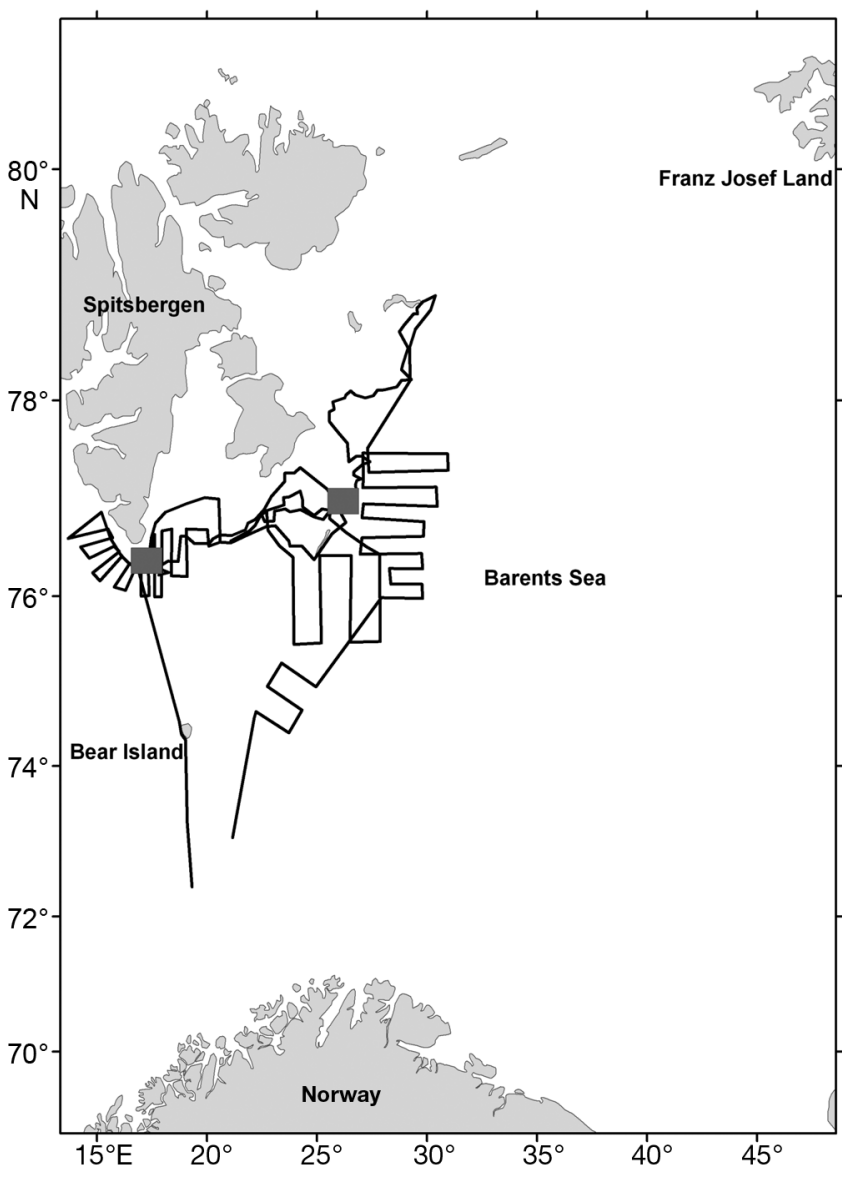

Fig. 1. Areas where harp seals Pagophilus groenlandicus were sampled (grey squares) and track lines for the acoustic survey performed by RV 'Jan Mayen' in May-June 2006. Several trawl hauls were made along the tracks for the resource mapping survey

items by conducting acoustic and trawl surveys in the assumed harp seal feeding areas. Sampling of seals and resource mapping were performed concurrently during the survey. In the areas south and southeast of Spitsbergen, very large numbers of seals were observed along the ice edge and 20 to 30 nautical miles south of this. No seals were observed in the surveyed open ice-free areas further away from the ice. In total, 57 harp seals were shot and sampled (Fig. 1). Additionally, samples of faeces were taken from the haul-out sites on the ice.

The seals were shot on ice floes and immediately brought onboard the vessel, where samples of digestive tracts were frozen for later examination of contents (Pettersen 2007). Dorsal blubber cores, $\sim 5 \times 5 \mathrm{~cm}$, were taken through the full depth of the blubber layer at the mid line between the front flippers. The cores were immediately wrapped in aluminium foil, packed in plastic bags and frozen until they could be transferred to the laboratory for analyses. Lower jaws with teeth were collected for age determination of all seals. One canine tooth was extracted after boiling the jaw. From each tooth, a 10-12 mm transverse section was mounted on a glass slide. Sections were examined under transmitted light and ages were estimated from counts of growth layers in the dentine (Bowen et al. 1983).

Estimation of potential prey abundance was carried out using standard acoustic survey methods (Lindstrøm et al. 1998) in the assumed feeding areas of the seals (Fig. 1). Both pelagic and demersal trawls were used to sample the observed acoustic scatterers. Based on harp seal diet observations made in the present and previous studies (Nilssen et al. 1995, Lindstrøm et al. 1998, 2006, Pettersen 2007), 16 different potential prey items were selected and sampled from the trawl hauls. Sufficient numbers of each of these items were frozen immediately for subsequent analyses of FA composition. The selected prey items included 4 crustacean species: large krill Meganyctiphanes norvegica, small krill Thysanoessa inermis, sympagic amphipods Gammarus wikitzkii and pelagic amphipods Parathemisto libellula. Furthermore, 12 fish species were selected for subsequent analyses: capelin Mallotus villosus, Arctic cod Gadus morhua, haddock Melanogrammus aeglefinus, Norway pout Trisopterus esmarki, polar cod Boreogadus saida, spotted snake blenny Lumpenus maculates, snake blenny Lumpenus lampraetiformis, Atlantic hooker sculpin Artediellus atlanticus, Atlantic poacher Leptagonus decagonus, eelpout Lycodes sp., long rough dab Hippoglossoides platessoides and Greenland halibut Reinhardtius hippoglossoides.

Laboratory methods. Subsamples were cut from the stored blubber samples while they were still frozen to avoid cross contamination. First, an outer slice through the whole sample from muscle to skin, $\sim 1 \mathrm{~cm}$ thick, was cut away to avoid lipids that might have been oxidized during storage. A rectangular core $(\sim 3 \times 3 \mathrm{~mm})$ of the exposed blubber was then cut out through the whole blubber core from muscle to skin. From this blubber core, $\sim 2 \mathrm{~mm}$ thick slices, weighing between 20 and $50 \mathrm{mg}$, were cut from the top and bottom, i.e. just above the muscles and just below the skin. From 4 of the seals, $2 \mathrm{~mm}$ sections were cut consecutively from the whole blubber core, from muscle to skin, resulting in 8 to 13 samples from each animal because of different thickness of their blubber.

The samples of potential prey species were thoroughly homogenised before retrieval of subsamples $\sim 50 \mathrm{mg}$ in weight. For large krill, sympagic amphipods and pelagic amphipods, 10 specimens, with a total weight of $\sim 3$ to $4 \mathrm{~g}$, were pooled and homogenised for each sample; for small krill, 25 specimens of similar weight were pooled and homogenised. Six parallel samples for each species were processed. For the 12 fish species, single individuals, between 2 and $>50 \mathrm{~g}$, 
were homogenised. Ten parallel fish were processed from each species except for snake blenny (9), spotted snake blenny (6) and Atlantic hooker sculpin (6).

The subsamples of seal blubber and potential prey were immediately transferred to tared $15 \mathrm{ml}$ thickwalled glass vials with Teflon-lined screwcaps, and their mass was accurately determined. Beforehand, $50.0 \mu \mathrm{l}$ of a chloroform (Puriss p.a., 99.0 to $99.4 \%$, Sigma-Aldrich) solution with an accurately determined concentration of the 19:0 FA methyl ester (FAME) had been added to the vials, and the chloroform was evaporated. This left an accurately known amount of 19:0 FAME as internal standard in each tube, $1.004 \mathrm{mg}$ for the blubber samples and $0.061 \mathrm{mg}$ for the potential prey samples. A volume of $0.6 \mathrm{ml}$ anhydrous methanol, containing hydrogen chloride $(\mathrm{HCl})$ with a concentration of $2 \mathrm{~mol} \mathrm{l}^{-1}$, was added to each vial. (The reagent was made by bubbling $\mathrm{HCl}$ gas - generated by letting concentrated hydrochloric acid [37\%, Merck] slowly into concentrated sulfuric acid [96\%, Merck]-into methanol [HPLC grade, 99.9\%, Sigma-Aldrich].) After exchange of the atmosphere in the tubes with nitrogen gas, the tubes were securely closed and placed in an oven for $2 \mathrm{~h}$ at $90^{\circ} \mathrm{C}$. After methanolysis, approximately half of the methanol/ $\mathrm{HCl}$ solution was evaporated using nitrogen gas, and $0.5 \mathrm{ml}$ of distilled water was added. The formed FAMEs were extracted twice with $1.0 \mathrm{ml}$ hexane (HPLC grade, Rathburn). The phases were mixed by vigorous shaking by hand for 1 min, followed by 6 min of mild centrifugation. The hexane phase was withdrawn using a Pasteur pipette. The 2 extracts were mixed. For the blubber samples, the mixed extracts were diluted with $2 \mathrm{ml}$ hexane.

For gas chromatography the extracts were diluted with hexane to obtain approximately the same response for all samples, i.e. 200 area units for 22:6n3. For the blubber samples, this meant that between 10 and $30 \mu \mathrm{l}$ of the extracts were diluted with $1 \mathrm{ml}$ hexane, and for the prey samples between 50 and $200 \mu \mathrm{l}$ were diluted with $0.5 \mathrm{ml}$ hexane. One $\mu \mathrm{l}$ of the dilutions was injected splitless and chromatographed on a HewlettPackard 5890A gas chromatograph equipped with a Hewlett-Packard 7673A autosampler and a flame ionisation detector. The column was $25 \mathrm{~m} \times 0.25 \mathrm{~mm}$ (i.d.) fused silica coated with polyethylene-glycol (CP-WAX 52CB Chrompack) of $0.2 \mu \mathrm{m}$ thickness, and helium was used as the mobile phase $\left(20 \mathrm{psi}\right.$, i.e. $\left.1.38 \times 10^{-5} \mathrm{~Pa}\right)$. The injector temperature was set at $260^{\circ} \mathrm{C}$. After injection, the temperature of the column was kept at $90^{\circ} \mathrm{C}$ for $4 \mathrm{~min}$ and then increased from $90^{\circ} \mathrm{C}$ to $165^{\circ} \mathrm{C}$ at a rate of $30^{\circ} \mathrm{C} \mathrm{min}{ }^{-1}$ followed by an increase of $3^{\circ} \mathrm{C}$ $\min ^{-1}$ to $225^{\circ} \mathrm{C}$. This temperature was maintained for $10.5 \mathrm{~min}$. The temperature of the flame ionisation detector was set at $330^{\circ} \mathrm{C}$. Samples were analysed in random order with a standard solution (GLC-68D,
Nu-Chek-Prep) containing 20 FAMEs between every eighth sample. The detector output was A/D converted, recorded, stored and treated using Chromeleon 6.60 software.

Forty-eight well-defined peaks in the chromatograms were selected. They were identified by comparison with a chromatogram of the standard mixture and chromatograms from previous analyses of marine FAMEs, and named according to the shorthand notation: (carbon chain length):(number of double bonds) $\mathrm{nX}$, where $\mathrm{nX}$ designates the position of the double bond nearest to the terminal methyl group.

Cholesterol was determined from 2 peaks, representing its decomposition products formed during methanolysis, appearing in the chromatograms with retention times of 34.66 and $35.15 \mathrm{~min}$, respectively (Meier et al. 2006). The response factor of these 2 peaks combined was $0.27 \pm 0.02($ mean $\pm \mathrm{SD})$ relative to 19:0 (Kwetegyeka et al. 2008).

Statistical analysis. The areas of the selected peaks were corrected by response factors. Empirical response factors, relative to $18: 0$, were obtained for the 20 FAMEs present in known proportions in the standard mixture. The response factors for each of the 12 FAMEs for which we had no standards were estimated by comparison with the standard FAMEs that resembled each of those most closely in terms of chain length and number of double bonds. The corrected values where then normalised to give the relative amount of each FA in each sample as a percentage of the sum of all FAs in the sample.

To obtain the combined information from all FAs simultaneously, the data were subjected to multivariate treatment based on principal component analysis (PCA). Fourteen of the 48 total FAs were not included in the PCA because they occurred in amounts $<0.1 \%$ of the total, and contributed more noise, i.e. random variation due to arbitrary integration of small peaks on top of a rugged (relatively) baseline, than real information to the results. The relative values (i.e. percent of the sum) of the FAs were scaled by dividing each value by the mean of the values of all samples for that particular FA. Thereby the quantitative differences among the FAs were levelled out, leaving them all to vary around one. With each sample positioned in the 34dimensional space described by the mean-scaled variables (FAs), new coordinates (principal components [PCs]) through the centroid of the samples in the directions of the largest and second largest variance among the samples were computed using SIRIUS (Kvalheim \& Karstang 1987). In this manner, the relationship between the samples in the original 34 dimensions could be described in 2 dimensions, without loss of the systematic variation among the samples. The relative positions of the samples were displayed by projecting 
them on a plane defined by the 2 new coordinates, i.e. the principal components PC1 and PC2.

To determine the distance in the 34-dimensional space of all samples to the inner blubber, a PC model of the inner blubber was generated. Cross validation showed that 6 significant components described the model. The outer boundary of the model, with a significance level of $95 \%$, i.e. residual standard deviation (RSD), was 0.093 .

Partial least square (PLS) analysis was used to detect possible dependence of the blubber FA composition on the age of the harp seals. Age was included in the data matrix as a dependent variable. The first PLS coordinate was determined through the centroid of the samples in the direction that gave the best correlation between the FAs and harp seal age. The second PLS coordinate, perpendicular to the first, was in the direction of the second best correlation between the FAs and age. In this manner, a model based on the 2 PLS coordinates was obtained that described $69 \%$ of the variance along the dependent variable. The correlation between the FA composition and the age of the seals was then displayed in a plot of measured versus predicted age.

A similar PLS regression was used to determine whether blubber FA composition was dependent on harp seal gender, using a dependent variable with a value of 1 for males and 2 for females.

\section{RESULTS}

The seal blubber contained the common spectrum of marine FAs, with 48 of those present in relative amounts of $>0.1 \%$ given in Table 1 . Individual differences were large, as indicated by the large standard deviations. Nevertheless, the difference between the inner and outer blubber was significant for all but 5 of the FAs $(\log (\mathrm{p})<-2)$.

The total amount of lipid in the seal blubber, determined as the sum of the FAs, as FAMEs, was $\sim 70 \%$, with significantly higher amounts in the outer blubber (Table 1). For the potential prey species there was a 10fold variation among the species, small krill having the highest level of lipids (7.7\%) and long rough dab being the leanest ( $0.7 \%$ lipid; Table 1$)$. Cholesterol was present in all potential prey species, ranging from $0.04 \%$ in sympagic amphipods to $0.9 \%$ in polar cod (Table 1 ), but cholesterol was not detected in the seal blubber.

The difference in the FA levels of the inner and outer blubber layers was determined as a stratification index (Fig. 2). This was obtained by subtracting the percentage in the inner layer from the percentage in the outer layer, as given in Table 1, and dividing the difference by the mean of the sum of the percentages in the 2 lay- ers. The differences between the 2 layers were quite systematic. All saturated FAs (SAFAs) were present in higher levels in the inner blubber. Of the monounsaturated FAs (MUFAs), the shorter ones (up to 18 carbons) had higher levels in the outer layer whereas the longer ones (20, 22 and 24 carbons) dominated in the inner layer. For the polyunsaturated FAs (PUFAs), the inner/outer differences were not as systematic as for the SAFAs and MUFAs, although the 3 FAs with 18 carbons all had higher levels in the outer layer.

The clear distinction between the layers, as well as the large individual differences, were illustrated by PCA, in which the 34 most abundant FAs (Table 1) were taken into account (Fig. 3). The sectioned samples from the 4 seals indicate that there was a more or less continuous transition in FA composition of the blubber from the inner to the outer layer (Fig. 4).

A distinction in FA composition of the blubber between genders was not evident, even if the most powerful tool for detection of differences between groups of samples, PLS, was applied to the data. Samples from the 2 genders overlapped in terms of FA composition, although not completely, and less completely for the outer layer (data not shown).

For the outer blubber there was a reasonably good correlation between age and FA composition $\left(\mathrm{R}^{2}=\right.$ 0.797; $\mathrm{p}=6 \times 10^{-7} ;$ Fig. 5). However, for the inner blubber the correlation was not significant $\left(R^{2}=0.315, p=\right.$ 0.06).

Evaluating each FA independently, large differences between the inner blubber layer and potential prey species from the trawl hauls were evident; pelagic amphipods had 29 significantly different FAs $(\log (\mathrm{p})$ $<-2$ ), and the number increased for the other prey species, up to 47 significantly different FAs for long rough dab (Table 1). When all FAs were evaluated simultaneously by PCA, the differences between the potential prey species and the blubber appeared to be very variable. Of the potential prey species, the FA composition of Parathemisto libellula, Thysanoessa inermis, Greenland halibut, Meganyctiphanes norvegica, Arctic cod, Gammarus wikitzkii and polar cod was quite similar to that of the inner blubber layer, with increasing differences for capelin, haddock, Norway pout, Atlantic poacher, Atlantic hooker sculpin, eelpout, spotted snake blenny, long rough dab and snake blenny (Fig. 6). This PC plot is, however, only a qualitative display, with all samples projected onto the plane of the first $2 \mathrm{PCs}$, covering two-thirds of the total variance among the samples.

A quantitative measure of the distances in the 34-dimensional space between the inner blubber and the prey was obtained by creating a PC model of the inner blubber samples and measuring how far outside this model the potential prey samples from the trawl 
Table 1. Fatty acids (FAs) in the inner and outer blubber of harp seals (Pagophilus groenlandicus) and in 16 prey species. Data are mean relative amounts (\% of sum $\pm \mathrm{SD}$ ). The logarithms of the p-values from $t$-testing of the amounts of the FA in the outer blubber and in the prey versus those in the inner blubber are given. M.: Meganyctiphanes; T.: Thysanoessa; P.: Parathemisto; G.: Gammarus. I.: inner; O.: outer; MUFA: monounsaturated FA; PUFA: polyunsaturated FA; SAFA: saturated FA; nd: not detected

\begin{tabular}{|c|c|c|c|c|c|c|c|c|c|c|c|c|c|c|c|c|c|}
\hline \multirow[t]{2}{*}{ Fatty acids } & \multirow{2}{*}{$\begin{array}{l}\text { I. blubber } \\
(\mathrm{N}=57) \\
\text { Mean } \\
\pm \mathrm{SD}\end{array}$} & \multicolumn{2}{|c|}{$\begin{array}{l}\text { O. blubber } \\
(\mathrm{N}=57)\end{array}$} & \multicolumn{2}{|c|}{$\begin{array}{l}\text { M. norvegica } \\
\quad(\mathrm{N}=6)\end{array}$} & \multicolumn{2}{|c|}{$\begin{array}{l}\text { T. inermis } \\
(\mathrm{N}=6)\end{array}$} & \multicolumn{2}{|c|}{$\begin{array}{l}\text { G. wikitzkii } \\
(\mathrm{N}=6)\end{array}$} & \multicolumn{2}{|c|}{$\begin{array}{l}\text { P. libellula } \\
(\mathrm{N}=6)\end{array}$} & \multicolumn{2}{|c|}{$\begin{array}{l}\text { Capelin } \\
(\mathrm{N}=10)\end{array}$} & \multicolumn{2}{|c|}{$\begin{array}{l}\text { Arctic cod } \\
(\mathrm{N}=10)\end{array}$} & \multicolumn{2}{|c|}{$\begin{array}{l}\text { Haddock } \\
(\mathrm{N}=10)\end{array}$} \\
\hline & & $\begin{array}{l}\text { Mean } \\
\pm \mathrm{SD}\end{array}$ & $\begin{array}{l}\log \\
(p)\end{array}$ & $\begin{array}{l}\text { Mean } \\
\pm \text { SD }\end{array}$ & $\begin{array}{l}\log \\
\text { (p) }\end{array}$ & $\begin{array}{l}\text { Mean } \\
\pm \text { SD }\end{array}$ & $\begin{array}{l}\log \\
\text { (p) }\end{array}$ & $\begin{array}{l}\text { Mean } \\
\pm \text { SD }\end{array}$ & $\begin{array}{l}\log \\
\text { (p) }\end{array}$ & $\begin{array}{l}\text { Mean } \\
\pm \text { SD }\end{array}$ & $\begin{array}{l}\log \\
\text { (p) }\end{array}$ & $\begin{array}{l}\text { Mean } \\
\pm \text { SD }\end{array}$ & $\begin{array}{l}\log \\
\text { (p) }\end{array}$ & $\begin{array}{l}\text { Mean } \\
\pm \text { SD }\end{array}$ & $\begin{array}{l}\log \\
\text { (p) }\end{array}$ & $\begin{array}{l}\text { Mean } \\
\pm \text { SD }\end{array}$ & $\begin{array}{l}\log \\
\text { (p) }\end{array}$ \\
\hline $14: 0$ & $5.8 \pm 0.6$ & $3.2 \pm 0.7$ & -53 & $5.1 \pm 0.1$ & -3 & $1.9 \pm 0.1$ & -27 & $3.6 \pm 0.1$ & -14 & $2.8 \pm 0.3$ & -21 & $4.8 \pm 1.7$ & -4 & $3.0 \pm 0.4$ & -25 & $2.8 \pm 0.6$ & -27 \\
\hline $14: 1 \mathrm{n} 5^{\mathrm{a}}$ & $0.8 \pm 0.2$ & $2.1 \pm 0.5$ & -43 & $0.1 \pm 0.0$ & -8 & $0.1 \pm 0.1$ & -9 & $0.1 \pm 0.0$ & -8 & $0.1 \pm 0.0$ & -9 & $0.1 \pm 0.0$ & -14 & $0.1 \pm 0.0$ & -14 & $0.0 \pm 0.0$ & -14 \\
\hline i- $15: 0$ & $0.3 \pm 0.0$ & $0.2 \pm 0.0$ & -32 & $0.3 \pm 0.0$ & -1 & $0.1 \pm 0.0$ & -13 & $0.1 \pm 0.0$ & -13 & $0.2 \pm 0.0$ & -6 & $0.3 \pm 0.1$ & 0 & $0.2 \pm 0.0$ & -16 & $0.2 \pm 0.1$ & -8 \\
\hline ai- $15: 0^{\mathrm{a}}$ & $0.1 \pm 0.0$ & $0.1 \pm 0.0$ & -19 & $0.1 \pm 0.0$ & -1 & $0.0 \pm 0.0$ & -16 & $0.0 \pm 0.0$ & -8 & $0.1 \pm 0.0$ & -5 & $0.1 \pm 0.0$ & -7 & $0.0 \pm 0.0$ & -18 & $0.0 \pm 0.0$ & -17 \\
\hline 15:0 & $0.3 \pm 0.1$ & $0.2 \pm 0.0$ & -28 & $0.4 \pm 0.0$ & -7 & $0.1 \pm 0.0$ & -11 & $0.3 \pm 0.0$ & -2 & $0.2 \pm 0.0$ & -4 & $0.5 \pm 0.1$ & -11 & $0.3 \pm 0.0$ & -2 & $0.4 \pm 0.1$ & -6 \\
\hline $15: 1 \mathrm{n} 5^{\mathrm{a}}$ & $0.0 \pm 0.0$ & $0.1 \pm 0.0$ & -53 & $0.0 \pm 0.0$ & -6 & $0.1 \pm 0.1$ & -20 & $0.0 \pm 0.0$ & 0 & $0.1 \pm 0.0$ & -10 & $0.0 \pm 0.0$ & -8 & $0.0 \pm 0.0$ & -14 & $0.0 \pm 0.0$ & -11 \\
\hline$i-16: 0^{a}$ & $0.1 \pm 0.0$ & $0.1 \pm 0.0$ & 0 & $0.1 \pm 0.0$ & -3 & $0.0 \pm 0.0$ & -20 & $0.1 \pm 0.0$ & -7 & $0.1 \pm 0.0$ & -5 & $0.1 \pm 0.0$ & -4 & $0.1 \pm 0.1$ & 0 & $0.1 \pm 0.1$ & -1 \\
\hline 16:0 & $7.9 \pm 1.2$ & $4.2 \pm 1.9$ & -28 & $14.8 \pm 0.6$ & -22 & $19.1 \pm 0.3$ & -35 & $11.6 \pm 1.0$ & -10 & $14.7 \pm 0.6$ & -22 & $15.3 \pm 1.4$ & -30 & $18.4 \pm 0.8$ & -42 & $14.8 \pm 1.6$ & -27 \\
\hline $16: 1 \mathrm{n} 11^{\mathrm{a}}$ & $0.1 \pm 0.0$ & $0.3 \pm 0.1$ & -44 & $0.0 \pm 0.0$ & -12 & $0.1 \pm 0.1$ & 0 & $0.1 \pm 0.0$ & -1 & $0.0 \pm 0.0$ & -13 & $0.1 \pm 0.0$ & -2 & $0.1 \pm 0.0$ & 0 & $0.2 \pm 0.0$ & -25 \\
\hline $16: 1 \mathrm{n} 9$ & $0.2 \pm 0.0$ & $0.4 \pm 0.1$ & -40 & $0.1 \pm 0.0$ & -14 & $0.0 \pm 0.0$ & -29 & $0.0 \pm 0.0$ & -26 & $0.1 \pm 0.0$ & -23 & $0.3 \pm 0.1$ & -1 & $0.2 \pm 0.0$ & 0 & $0.3 \pm 0.1$ & -3 \\
\hline $16: 1 \mathrm{n} 7$ & $9.9 \pm 1.8$ & $17.8 \pm 2.2$ & -50 & $12.7 \pm 0.6$ & -3 & $17.6 \pm 0.5$ & -16 & $17.9 \pm 1.0$ & -16 & $12.1 \pm 0.5$ & -2 & $5.1 \pm 1.1$ & -12 & $7.5 \pm 0.6$ & -4 & $5.5 \pm 1.5$ & -10 \\
\hline $16: 1 \mathrm{n} 5$ & $0.3 \pm 0.0$ & $0.3 \pm 0.0$ & -9 & $0.3 \pm 0.0$ & -5 & $0.1 \pm 0.0$ & -10 & $0.2 \pm 0.0$ & -2 & $0.2 \pm 0.0$ & 0 & $0.4 \pm 0.1$ & -15 & $0.2 \pm 0.0$ & -1 & $0.2 \pm 0.0$ & -1 \\
\hline i- $17: 0$ & $0.2 \pm 0.0$ & $0.2 \pm 0.0$ & -6 & $0.4 \pm 0.0$ & -21 & $0.1 \pm 0.0$ & -12 & $0.2 \pm 0.0$ & 0 & $0.2 \pm 0.0$ & -6 & $0.5 \pm 0.1$ & -33 & $0.2 \pm 0.0$ & -3 & $0.5 \pm 0.1$ & -31 \\
\hline ai- $17: 0^{\mathrm{a}}$ & $0.1 \pm 0.0$ & $0.1 \pm 0.0$ & -1 & $0.1 \pm 0.0$ & -5 & $0.0 \pm 0.0$ & -20 & $0.1 \pm 0.0$ & -6 & $0.1 \pm 0.0$ & -2 & $0.1 \pm 0.0$ & -10 & $0.1 \pm 0.0$ & -3 & $0.2 \pm 0.0$ & -18 \\
\hline $16: 2 n x$ & $0.5 \pm 0.1$ & $0.6 \pm 0.1$ & -9 & $0.7 \pm 0.0$ & -3 & $1.0 \pm 0.0$ & -12 & $0.8 \pm 0.1$ & -6 & $0.6 \pm 0.0$ & -1 & $0.3 \pm 0.1$ & -6 & $0.4 \pm 0.0$ & -2 & $0.3 \pm 0.1$ & -7 \\
\hline $16: 2 \mathrm{n} 6$ & $0.2 \pm 0.0$ & $0.1 \pm 0.0$ & -15 & $0.0 \pm 0.0$ & -18 & $0.0 \pm 0.0$ & -18 & $0.0 \pm 0.0$ & -17 & $0.0 \pm 0.0$ & -18 & $0.4 \pm 0.1$ & -18 & $0.1 \pm 0.0$ & -9 & $0.3 \pm 0.0$ & -18 \\
\hline Unid. $^{a}$ & $0.2 \pm 0.1$ & $0.1 \pm 0.0$ & -16 & $0.6 \pm 0.1$ & -25 & $0.0 \pm 0.0$ & -4 & $0.5 \pm 0.1$ & -13 & $0.1 \pm 0.0$ & -2 & $0.3 \pm 0.1$ & -5 & $1.3 \pm 0.1$ & -57 & $0.6 \pm 0.2$ & -23 \\
\hline $17: 0^{\mathrm{a}}$ & $0.1 \pm 0.0$ & $0.0 \pm 0.0$ & -25 & $0.1 \pm 0.0$ & 0 & $0.0 \pm 0.0$ & -7 & $0.1 \pm 0.0$ & -3 & $0.1 \pm 0.0$ & -2 & $0.2 \pm 0.0$ & -5 & $0.1 \pm 0.0$ & -1 & $0.3 \pm 0.0$ & -17 \\
\hline 17:1n11 & $0.2 \pm 0.1$ & $0.2 \pm 0.0$ & -7 & $0.7 \pm 0.1$ & -21 & $0.7 \pm 0.1$ & -19 & $0.7 \pm 0.1$ & -18 & $0.3 \pm 0.0$ & -1 & $0.2 \pm 0.1$ & -1 & $0.1 \pm 0.0$ & -4 & $0.1 \pm 0.1$ & -4 \\
\hline $17: 1 \mathrm{n} 9$ & $0.2 \pm 0.1$ & $0.3 \pm 0.1$ & -16 & $0.3 \pm 0.0$ & -1 & $0.1 \pm 0.0$ & -5 & $0.1 \pm 0.0$ & -3 & $0.2 \pm 0.0$ & -2 & $0.3 \pm 0.1$ & -2 & $0.2 \pm 0.0$ & -1 & $0.3 \pm 0.1$ & -3 \\
\hline $16: 4 \mathrm{n} 1$ & $0.3 \pm 0.1$ & $0.3 \pm 0.1$ & -1 & $1.0 \pm 0.1$ & -23 & $0.5 \pm 0.1$ & -5 & $1.2 \pm 0.1$ & -30 & $0.3 \pm 0.0$ & 0 & $0.4 \pm 0.2$ & 0 & $0.2 \pm 0.0$ & -6 & $0.2 \pm 0.1$ & -4 \\
\hline 18:0 & $1.1 \pm 0.2$ & $0.4 \pm 0.1$ & -49 & $1.3 \pm 0.1$ & -1 & $1.6 \pm 0.1$ & -8 & $0.7 \pm 0.1$ & -5 & $1.1 \pm 0.1$ & 0 & $2.1 \pm 0.5$ & -19 & $3.6 \pm 0.3$ & -51 & $3.9 \pm 0.5$ & -49 \\
\hline $18: 1 \mathrm{n} 11^{\mathrm{a}}$ & $0.0 \pm 0.0$ & $0.0 \pm 0.0$ & -1 & $0.0 \pm 0.0$ & -6 & $0.5 \pm 1.0$ & -6 & $0.0 \pm 0.0$ & 0 & $0.0 \pm 0.0$ & -4 & $0.0 \pm 0.0$ & -34 & $0.0 \pm 0.0$ & -6 & $0.1 \pm 0.1$ & -13 \\
\hline 18:1n9 & $16.9 \pm 1.8$ & $25.6 \pm 2.3$ & -54 & $10.0 \pm 0.3$ & -14 & $19.1 \pm 1.0$ & -2 & $17.3 \pm 1.2$ & 0 & $16.7 \pm 0.9$ & 0 & $9.1 \pm 1.2$ & -22 & $15.8 \pm 0.8$ & -1 & $11.5 \pm 0.9$ & -14 \\
\hline $18: 1 \mathrm{n} 7$ & $3.8 \pm 0.7$ & $4.7 \pm 0.7$ & -12 & $4.4 \pm 0.2$ & -1 & $8.8 \pm 0.3$ & -29 & $3.3 \pm 0.6$ & -1 & $6.6 \pm 0.4$ & -15 & $2.8 \pm 0.4$ & -5 & $7.4 \pm 0.5$ & -27 & $4.2 \pm 0.7$ & -1 \\
\hline $18: 1 \mathrm{n} 5$ & $0.5 \pm 0.1$ & $0.4 \pm 0.1$ & -8 & $0.5 \pm 0.0$ & -2 & $0.1 \pm 0.0$ & -21 & $0.6 \pm 0.1$ & -5 & $0.6 \pm 0.1$ & -8 & $0.6 \pm 0.1$ & -5 & $0.3 \pm 0.0$ & -14 & $0.4 \pm 0.1$ & -2 \\
\hline $18: 2 \mathrm{n} 6^{\mathrm{b}}$ & $1.5 \pm 0.1$ & $2.0 \pm 0.2$ & -34 & $1.0 \pm 0.0$ & -11 & $0.8 \pm 0.0$ & -18 & $1.0 \pm 0.0$ & -12 & $1.2 \pm 0.1$ & -5 & $1.3 \pm 0.2$ & -4 & $1.1 \pm 0.1$ & -11 & $1.0 \pm 0.2$ & -15 \\
\hline $18: 3 \mathrm{n} 3$ & $0.6 \pm 0.1$ & $0.9 \pm 0.1$ & -31 & $0.3 \pm 0.0$ & -6 & $0.2 \pm 0.1$ & -8 & $0.3 \pm 0.0$ & -6 & $0.4 \pm 0.1$ & -4 & $0.5 \pm 0.1$ & -1 & $0.6 \pm 0.1$ & 0 & $0.4 \pm 0.1$ & -4 \\
\hline $18: 4 \mathrm{n} 3^{b}$ & $1.8 \pm 0.5$ & $2.3 \pm 0.3$ & -12 & $2.0 \pm 0.1$ & -1 & $2.6 \pm 0.1$ & -4 & $2.3 \pm 0.2$ & -2 & $2.1 \pm 0.2$ & -1 & $1.3 \pm 0.3$ & -2 & $2.4 \pm 0.3$ & -4 & $1.1 \pm 0.4$ & -4 \\
\hline $20: 0^{\mathrm{a}}$ & $0.1 \pm 0.0$ & $0.0 \pm 0.0$ & -53 & $0.1 \pm 0.0$ & -4 & $0.0 \pm 0.0$ & -10 & $0.0 \pm 0.0$ & -7 & $0.0 \pm 0.0$ & -12 & $0.1 \pm 0.0$ & -1 & $0.0 \pm 0.0$ & -17 & $0.1 \pm 0.0$ & -7 \\
\hline $20: 1 \mathrm{n} 11^{\mathrm{b}}$ & $1.9 \pm 0.3$ & $1.5 \pm 0.3$ & -10 & $0.5 \pm 0.0$ & -15 & $0.1 \pm 0.1$ & -21 & $0.4 \pm 0.4$ & -15 & $1.4 \pm 0.5$ & -2 & $0.4 \pm 0.1$ & -22 & $0.4 \pm 0.1$ & -22 & $0.8 \pm 0.2$ & -16 \\
\hline $20: 1 \mathrm{n} 9^{\mathrm{b}}$ & $14.2 \pm 2.7$ & $7.1 \pm 1.5$ & -41 & $10.4 \pm 1.1$ & -3 & $0.3 \pm 0.3$ & -20 & $8.6 \pm 1.1$ & -6 & $8.5 \pm 1.7$ & -6 & $7.0 \pm 3.0$ & -11 & $2.9 \pm 0.6$ & -22 & $6.7 \pm 2.6$ & -12 \\
\hline $20: 1 \mathrm{n} 7^{\mathrm{b}}$ & $0.5 \pm 0.1$ & $0.2 \pm 0.0$ & -43 & $0.6 \pm 0.0$ & -4 & $0.0 \pm 0.0$ & -21 & $0.4 \pm 0.1$ & -1 & $0.4 \pm 0.1$ & -1 & $0.3 \pm 0.1$ & -10 & $0.2 \pm 0.0$ & -12 & $0.5 \pm 0.1$ & 0 \\
\hline $20: 2 \mathrm{n} 6$ & $0.3 \pm 0.0$ & $0.2 \pm 0.0$ & -30 & $0.3 \pm 0.1$ & 0 & $0.1 \pm 0.0$ & -27 & $0.1 \pm 0.0$ & -19 & $0.1 \pm 0.1$ & -13 & $0.2 \pm 0.1$ & -14 & $0.3 \pm 0.0$ & 0 & $0.3 \pm 0.1$ & -1 \\
\hline $20: 4 \mathrm{n} 6$ & $0.3 \pm 0.1$ & $0.4 \pm 0.1$ & -5 & $0.5 \pm 0.0$ & -7 & $0.3 \pm 0.0$ & -1 & $0.5 \pm 0.1$ & -5 & $0.3 \pm 0.0$ & 0 & $0.7 \pm 0.2$ & -23 & $0.8 \pm 0.1$ & -31 & $1.2 \pm 0.3$ & -34 \\
\hline $20: 3 \mathrm{n} 3^{\mathrm{a}}$ & $0.1 \pm 0.0$ & $0.1 \pm 0.0$ & -6 & $0.3 \pm 0.0$ & -51 & $0.0 \pm 0.0$ & -8 & $0.1 \pm 0.0$ & -5 & $0.1 \pm 0.0$ & 0 & $0.1 \pm 0.0$ & -1 & $0.1 \pm 0.0$ & -6 & $0.1 \pm 0.0$ & -9 \\
\hline $20: 4 \mathrm{n} 3^{\mathrm{b}}$ & $0.5 \pm 0.1$ & $0.6 \pm 0.1$ & -2 & $0.5 \pm 0.0$ & 0 & $0.2 \pm 0.0$ & -9 & $0.4 \pm 0.0$ & -1 & $0.3 \pm 0.0$ & -5 & $0.5 \pm 0.1$ & 0 & $0.4 \pm 0.1$ & -3 & $0.4 \pm 0.1$ & -3 \\
\hline $20: 5 n 3^{b}$ & $4.1 \pm 1.2$ & $7.5 \pm 1.3$ & -33 & $11.7 \pm 0.8$ & -25 & $18.0 \pm 1.3$ & -42 & $11.6 \pm 0.4$ & -25 & $13.1 \pm 0.8$ & -30 & $11.7 \pm 2.2$ & -29 & $14.1 \pm 0.6$ & -42 & $11.9 \pm 1.0$ & -33 \\
\hline $22: 1 \mathrm{n} 11^{\mathrm{b}}$ & $6.6 \pm 2.0$ & $0.9 \pm 0.6$ & -47 & $6.8 \pm 0.4$ & 0 & $0.2 \pm 0.0$ & -10 & $7.0 \pm 1.0$ & 0 & $3.9 \pm 0.8$ & -3 & $5.8 \pm 2.5$ & -1 & $1.3 \pm 0.2$ & -12 & $4.6 \pm 2.9$ & -2 \\
\hline $22: 1 \mathrm{n} 9^{b}$ & $1.0 \pm 0.2$ & $0.2 \pm 0.1$ & -53 & $1.0 \pm 0.1$ & 0 & $0.1 \pm 0.1$ & -12 & $1.3 \pm 0.2$ & -3 & $0.9 \pm 0.2$ & 0 & $0.6 \pm 0.2$ & -5 & $0.3 \pm 0.0$ & -13 & $0.8 \pm 0.2$ & -2 \\
\hline $22: 1 \mathrm{n} 7^{\mathrm{a}}$ & $0.1 \pm 0.0$ & $0.1 \pm 0.0$ & -47 & $0.2 \pm 0.0$ & -7 & $0.2 \pm 0.0$ & 0 & $0.2 \pm 0.0$ & -4 & $0.2 \pm 0.0$ & -4 & $0.2 \pm 0.0$ & 0 & $0.1 \pm 0.0$ & -2 & $0.1 \pm 0.1$ & 0 \\
\hline $21: 5 \mathrm{n} 3$ & $0.4 \pm 0.1$ & $0.5 \pm 0.1$ & -6 & $0.4 \pm 0.0$ & 0 & $0.3 \pm 0.0$ & -4 & $0.3 \pm 0.0$ & -2 & $0.2 \pm 0.0$ & -5 & $0.3 \pm 0.0$ & -2 & $0.5 \pm 0.1$ & 0 & $0.3 \pm 0.1$ & -4 \\
\hline $22: 4 \mathrm{n} 6$ & $0.2 \pm 0.1$ & $0.1 \pm 0.0$ & -26 & $0.1 \pm 0.0$ & -5 & $0.0 \pm 0.0$ & -8 & $0.1 \pm 0.0$ & -6 & $0.1 \pm 0.0$ & -2 & $0.3 \pm 0.1$ & -3 & $0.1 \pm 0.0$ & -3 & $0.2 \pm 0.0$ & 0 \\
\hline $22: 5 n 6$ & $0.1 \pm 0.0$ & $0.1 \pm 0.0$ & -13 & $0.1 \pm 0.0$ & 0 & $0.1 \pm 0.1$ & -1 & $0.1 \pm 0.0$ & -4 & $0.0 \pm 0.0$ & -6 & $0.2 \pm 0.0$ & -3 & $0.1 \pm 0.0$ & -3 & $0.4 \pm 0.1$ & -31 \\
\hline $22: 5 \mathrm{n} 3$ & $5.0 \pm 1.2$ & $4.4 \pm 0.8$ & -3 & $0.5 \pm 0.0$ & -13 & $0.1 \pm 0.1$ & -15 & $0.3 \pm 0.0$ & -14 & $0.3 \pm 0.0$ & -14 & $1.0 \pm 0.2$ & -16 & $0.6 \pm 0.1$ & -18 & $1.0 \pm 0.1$ & -16 \\
\hline $24: 0^{\mathrm{a}}$ & $0.0 \pm 0.0$ & $0.0 \pm 0.0$ & -6 & $0.0 \pm 0.0$ & -3 & $0.1 \pm 0.1$ & -2 & $0.0 \pm 0.0$ & -3 & $0.0 \pm 0.0$ & -4 & $0.1 \pm 0.1$ & -16 & $0.0 \pm 0.0$ & -1 & $0.1 \pm 0.1$ & -9 \\
\hline $22: 6 n 3^{b}$ & $10.1 \pm 1.4$ & $8.7 \pm 1.3$ & -8 & $7.9 \pm 0.6$ & -4 & $4.1 \pm 0.4$ & -17 & $4.7 \pm 0.2$ & -14 & $8.5 \pm 0.5$ & -2 & $21.4 \pm 4.6$ & -28 & $13.2 \pm 1.6$ & -8 & $19.2 \pm 4.7$ & -22 \\
\hline $24: 1 \mathrm{n} 9$ & $0.4 \pm 0.1$ & $0.1 \pm 0.1$ & -36 & $0.6 \pm 0.1$ & -3 & $0.1 \pm 0.1$ & -5 & $0.6 \pm 0.1$ & -2 & $0.4 \pm 0.0$ & 0 & $2.1 \pm 0.6$ & -36 & $0.8 \pm 0.2$ & -10 & $1.6 \pm 0.4$ & -32 \\
\hline$\Sigma$ SAFA & 16.2 & 8.8 & & 22.8 & & 23.2 & & 16.9 & & 19. & & 24 & & 26 & & 23.2 & \\
\hline$\Sigma$ MUFA & 57.6 & 62.4 & & 49.3 & & 48.4 & & 59.0 & & 52.7 & & 35.2 & & 37.9 & & 38.0 & \\
\hline$\Sigma$ PUFA & 26.0 & 28.7 & & 27.3 & & 28.3 & & 23.7 & & 27.7 & & 40.4 & & 34.8 & & 38.2 & \\
\hline$\%$ total FA & $71 \pm 8$ & $76 \pm 5$ & -3 & $5.3 \pm 0.0$ & & $7.7 \pm 0.7$ & & $3.2 \pm 0.0$ & & $2.90 \pm 0.0$ & & $1.9 \pm 0.8$ & & $3.0 \pm 0.6$ & & $1.60 \pm 0.2$ & \\
\hline$\%$ cholester & rol nd & nd & & $0.06 \pm 0.0$ & & $0.05 \pm 0.0$ & & $0.04 \pm 0.0$ & & $0.62 \pm 0.0$ & & $0.75 \pm 0.0$ & & $0.60 \pm 0.0$ & & $0.17 \pm 0.0$ & \\
\hline
\end{tabular}


Table 1 (continued)

\begin{tabular}{|c|c|c|c|c|c|c|c|c|c|c|c|c|c|c|c|c|c|c|}
\hline \multirow[t]{2}{*}{ Fatty acids } & \multicolumn{2}{|c|}{$\begin{array}{c}\text { Norway } \\
\text { pout } \\
(\mathrm{N}=10)\end{array}$} & \multicolumn{2}{|c|}{$\begin{array}{l}\text { Polar cod } \\
(\mathrm{N}=10)\end{array}$} & \multicolumn{2}{|c|}{$\begin{array}{c}\text { Spotted } \\
\text { snake blenny } \\
(\mathrm{N}=6)\end{array}$} & \multicolumn{2}{|c|}{$\begin{array}{l}\text { Snake } \\
\text { blenny } \\
(\mathrm{N}=9)\end{array}$} & \multicolumn{2}{|c|}{$\begin{array}{c}\text { Atlantic } \\
\text { hooker sculpin } \\
(\mathrm{N}=6)\end{array}$} & \multicolumn{2}{|c|}{$\begin{array}{l}\text { Atlantic } \\
\text { poacher } \\
(\mathrm{N}=10)\end{array}$} & \multicolumn{2}{|c|}{$\begin{array}{l}\text { Eelpout } \\
(\mathrm{N}=10)\end{array}$} & \multicolumn{2}{|c|}{$\begin{array}{l}\text { Long } \\
\text { rough dab } \\
(\mathrm{N}=10)\end{array}$} & \multicolumn{2}{|c|}{$\begin{array}{l}\text { Greenland } \\
\text { halibut } \\
(\mathrm{N}=10)\end{array}$} \\
\hline & $\begin{array}{l}\text { Mean } \\
\pm \mathrm{SD}\end{array}$ & $\begin{array}{l}\log \\
\text { (p) }\end{array}$ & $\begin{array}{c}\text { Mean } \\
\pm \text { SD }\end{array}$ & $\begin{array}{c}\log \\
(p)\end{array}$ & $\begin{array}{c}\text { Mean } \\
\pm \text { SD }\end{array}$ & $\begin{array}{c}\log \\
\text { (p) }\end{array}$ & $\begin{array}{l}\text { Mean } \\
\pm \mathrm{SD}\end{array}$ & $\begin{array}{l}\log \\
\text { (p) }\end{array}$ & $\begin{array}{c}\text { Mean } \\
\pm \text { SD }\end{array}$ & $\begin{array}{c}\log \\
\text { (p) }\end{array}$ & $\begin{array}{c}\text { Mean } \\
\pm \text { SD }\end{array}$ & $\begin{array}{c}\log \\
(p)\end{array}$ & $\begin{array}{c}\text { Mean } \\
\pm \text { SD }\end{array}$ & $\begin{array}{l}\log \\
\text { (p) }\end{array}$ & $\begin{array}{c}\text { Mean } \\
\pm \text { SD }\end{array}$ & $\begin{array}{c}\log \\
\text { (p) }\end{array}$ & $\begin{array}{c}\text { Mean } \\
\pm \text { SD }\end{array}$ & $\begin{array}{l}\text { Log } \\
\text { (p) }\end{array}$ \\
\hline $14: 0$ & $2.6 \pm 0.6$ & -28 & $3.4 \pm 0.9$ & -19 & $1.2 \pm 0.2$ & -32 & $2.0 \pm 0.3$ & -32 & $2.4 \pm 0.5$ & -23 & $3.1 \pm 0.4$ & -25 & $2.4 \pm 0.5$ & -24 & $1.6 \pm 0.2$ & -38 & $4.6 \pm 0.2$ & -9 \\
\hline $14: 1 \mathrm{n} 5^{\mathrm{a}}$ & $0.0 \pm 0.0$ & -14 & $0.0 \pm 0.0$ & -14 & $0.0 \pm 0.0$ & -10 & $0.1 \pm 0.0$ & -12 & $0.1 \pm 0.0$ & -9 & $0.2 \pm 0.0$ & -11 & $0.1 \pm 0.0$ & -8 & $0.0 \pm 0.0$ & -15 & $0.1 \pm 0.0$ & -13 \\
\hline i- $15: 0$ & $0.2 \pm 0.0$ & -13 & $0.2 \pm 0.1$ & -10 & $0.1 \pm 0.0$ & -14 & $0.6 \pm 0.2$ & -15 & $0.2 \pm 0.0$ & -3 & $0.3 \pm 0.0$ & -1 & $0.2 \pm 0.0$ & -8 & $0.2 \pm 0.0$ & -13 & $0.2 \pm 0.0$ & -12 \\
\hline ai- $15: 0^{a}$ & $0.0 \pm 0.0$ & -21 & $0.0 \pm 0.0$ & -12 & $0.0 \pm 0.0$ & -12 & $0.3 \pm 0.1$ & -20 & $0.1 \pm 0.0$ & -6 & $0.1 \pm 0.0$ & -11 & $0.1 \pm 0.1$ & 0 & $0.1 \pm 0.0$ & -5 & $0.0 \pm 0.0$ & -11 \\
\hline 15:0 & $0.4 \pm 0.1$ & -3 & $0.3 \pm 0.0$ & 0 & $0.4 \pm 0.0$ & -5 & $0.8 \pm 0.1$ & -34 & $0.4 \pm 0.0$ & -7 & $0.4 \pm 0.0$ & -5 & $0.4 \pm 0.1$ & -4 & $0.5 \pm 0.1$ & -13 & $0.3 \pm 0.0$ & 0 \\
\hline $15: 1 \mathrm{n} 5^{\mathrm{a}}$ & $0.0 \pm 0.0$ & -11 & $0.0 \pm 0.0$ & -11 & $0.0 \pm 0.0$ & -7 & $0.1 \pm 0.0$ & -7 & $0.0 \pm 0.0$ & 0 & $0.0 \pm 0.0$ & 0 & $0.0 \pm 0.0$ & -1 & $0.0 \pm 0.0$ & -10 & $0.0 \pm 0.0$ & -13 \\
\hline $\mathrm{i}-16: 0^{\mathrm{a}}$ & $0.1 \pm 0.0$ & -1 & $0.1 \pm 0.0$ & -6 & $0.2 \pm 0.0$ & -8 & $0.6 \pm 0.2$ & -35 & $0.2 \pm 0.0$ & -20 & $0.3 \pm 0.0$ & -40 & $0.2 \pm 0.1$ & -13 & $0.1 \pm 0.1$ & -1 & $0.1 \pm 0.0$ & -3 \\
\hline 16:0 & $15.5 \pm 1.6$ & -30 & $14.2 \pm 2.6$ & -21 & $14.1 \pm 0.9$ & -19 & $13.3 \pm 1.3$ & -20 & $12.9 \pm 0.7$ & -15 & $13.4 \pm 0.9$ & -23 & $14.0 \pm 1.9$ & -17 & $16.5 \pm 0.7$ & -36 & $20.0 \pm 0.5$ & -47 \\
\hline $16: 1 \mathrm{n} 11^{\mathrm{a}}$ & $0.2 \pm 0.1$ & -23 & $0.1 \pm 0.0$ & -3 & $0.2 \pm 0.1$ & -9 & $0.1 \pm 0.0$ & -2 & $0.2 \pm 0.0$ & -21 & $0.3 \pm 0.0$ & -32 & $0.3 \pm 0.1$ & -25 & $0.5 \pm 0.1$ & -40 & $0.1 \pm 0.0$ & -4 \\
\hline $16: 1 \mathrm{n} 9$ & $0.4 \pm 0.1$ & -20 & $0.3 \pm 0.1$ & -7 & $0.5 \pm 0.1$ & -23 & $0.8 \pm 0.2$ & -43 & $0.4 \pm 0.2$ & -10 & $0.5 \pm 0.1$ & -22 & $0.6 \pm 0.1$ & -33 & $0.8 \pm 0.2$ & -36 & $0.2 \pm 0.0$ & 0 \\
\hline $16: \ln 7$ & $5.9 \pm 1.2$ & -9 & $8.3 \pm 2.1$ & -2 & $5.1 \pm 3.0$ & -7 & $9.1 \pm 2.3$ & -1 & $7.7 \pm 0.6$ & -2 & $8.9 \pm 1.2$ & -1 & $8.8 \pm 0.8$ & -1 & $2.2 \pm 0.2$ & -22 & $8.6 \pm 0.7$ & -2 \\
\hline $16: 1 \mathrm{n} 5$ & $0.2 \pm 0.0$ & -1 & $0.4 \pm 0.1$ & -13 & $0.3 \pm 0.0$ & -6 & $1.0 \pm 0.2$ & -39 & $0.4 \pm 0.0$ & -13 & $0.4 \pm 0.1$ & -18 & $0.4 \pm 0.1$ & -9 & $0.5 \pm 0.1$ & -24 & $0.3 \pm 0.0$ & -3 \\
\hline i-17:0 & $0.4 \pm 0.1$ & -26 & $0.3 \pm 0.1$ & -4 & $1.4 \pm 0.1$ & -74 & $1.4 \pm 0.2$ & -62 & $0.7 \pm 0.1$ & -51 & $0.5 \pm 0.0$ & -45 & $0.6 \pm 0.1$ & -34 & $1.2 \pm 0.3$ & -42 & $0.2 \pm 0.0$ & -1 \\
\hline ai- $17: 0^{a}$ & $0.2 \pm 0.0$ & -26 & $0.1 \pm 0.0$ & -3 & $0.3 \pm 0.1$ & -45 & $0.9 \pm 0.2$ & -47 & $0.3 \pm 0.0$ & -40 & $0.3 \pm 0.0$ & -56 & $0.3 \pm 0.1$ & -36 & $0.4 \pm 0.1$ & -51 & $0.1 \pm 0.0$ & -4 \\
\hline $16: 2 \mathrm{nx}$ & $0.3 \pm 0.0$ & -7 & $0.5 \pm 0.2$ & 0 & $0.1 \pm 0.0$ & -11 & $0.2 \pm 0.1$ & -9 & $0.3 \pm 0.1$ & -4 & $0.3 \pm 0.1$ & -7 & $0.3 \pm 0.1$ & -4 & $0.1 \pm 0.0$ & -17 & $0.4 \pm 0.0$ & -2 \\
\hline $16: 2 \mathrm{n} 6$ & $0.3 \pm 0.1$ & -11 & $0.2 \pm 0.1$ & -4 & $1.2 \pm 0.1$ & -66 & $0.9 \pm 0.3$ & -32 & $0.4 \pm 0.1$ & -20 & $0.3 \pm 0.0$ & -11 & $0.5 \pm 0.1$ & -28 & $1.1 \pm 0.2$ & -46 & $0.1 \pm 0.0$ & -8 \\
\hline Unid. ${ }^{a}$ & $0.7 \pm 0.3$ & -20 & $0.4 \pm 0.2$ & -7 & $0.1 \pm 0.1$ & -1 & $0.3 \pm 0.2$ & -5 & $0.2 \pm 0.1$ & -1 & $0.3 \pm 0.2$ & -7 & $0.5 \pm 0.3$ & -8 & $0.1 \pm 0.1$ & -6 & $1.5 \pm 0.2$ & -60 \\
\hline $17: 0^{\mathrm{a}}$ & $0.2 \pm 0.1$ & -12 & $0.1 \pm 0.0$ & 0 & $0.4 \pm 0.1$ & -24 & $0.8 \pm 0.2$ & -44 & $0.4 \pm 0.0$ & -23 & $0.3 \pm 0.0$ & -15 & $0.4 \pm 0.1$ & -17 & $0.4 \pm 0.1$ & -26 & $0.1 \pm 0.0$ & -3 \\
\hline 17:1n11 & $0.1 \pm 0.1$ & -3 & $0.2 \pm 0.1$ & -2 & $0.0 \pm 0.0$ & -8 & $0.1 \pm 0.1$ & -4 & $0.1 \pm 0.1$ & -3 & $0.0 \pm 0.0$ & -10 & $0.1 \pm 0.1$ & -6 & $0.0 \pm 0.0$ & -12 & $0.1 \pm 0.0$ & -6 \\
\hline $17: 1 \mathrm{n} 9$ & $0.3 \pm 0.1$ & -4 & $0.2 \pm 0.0$ & -1 & $0.5 \pm 0.0$ & -12 & $0.9 \pm 0.1$ & -39 & $0.5 \pm 0.0$ & -11 & $0.4 \pm 0.1$ & -10 & $0.6 \pm 0.2$ & -14 & $0.5 \pm 0.2$ & -11 & $0.2 \pm 0.0$ & -1 \\
\hline $16: 4 \mathrm{n} 1$ & $0.2 \pm 0.1$ & -4 & $0.3 \pm 0.2$ & 0 & $0.3 \pm 0.2$ & -1 & $0.3 \pm 0.1$ & 0 & $0.3 \pm 0.1$ & -1 & $0.1 \pm 0.1$ & -7 & $0.2 \pm 0.1$ & -3 & $0.2 \pm 0.1$ & -3 & $0.2 \pm 0.0$ & -6 \\
\hline $18: 0$ & $4.0 \pm 0.4$ & -54 & $2.4 \pm 0.6$ & -23 & $4.4 \pm 0.7$ & -46 & $4.1 \pm 0.5$ & -52 & $3.3 \pm 0.3$ & -39 & $2.7 \pm 0.6$ & -29 & $4.1 \pm 0.3$ & -49 & $5.8 \pm 0.3$ & -73 & $2.6 \pm 0.1$ & -38 \\
\hline $18: 1 \mathrm{n} 11^{\mathrm{a}}$ & $0.1 \pm 0.1$ & -16 & $0.0 \pm 0.0$ & -4 & $0.9 \pm 0.1$ & -72 & $0.9 \pm 0.1$ & -71 & $0.6 \pm 0.2$ & -53 & $0.2 \pm 0.1$ & -29 & $0.4 \pm 0.3$ & -22 & $0.3 \pm 0.2$ & -29 & $0.0 \pm 0.0$ & -4 \\
\hline $18: 1 \mathrm{n} 9$ & $12.1 \pm 1.0$ & -12 & $10.7 \pm 2.1$ & -16 & $9.6 \pm 2.6$ & -14 & $8.9 \pm 1.6$ & -21 & $14.8 \pm 0.7$ & -2 & $14.8 \pm 0.6$ & -3 & $13.8 \pm 1.0$ & -4 & $7.7 \pm 0.9$ & -27 & $16.2 \pm 0.9$ & -1 \\
\hline $18: 1 \mathrm{n} 7$ & $4.6 \pm 0.7$ & -3 & $3.9 \pm 1.2$ & 0 & $5.4 \pm 0.6$ & -6 & $6.9 \pm 1.0$ & -20 & $7.1 \pm 0.8$ & -17 & $7.5 \pm 0.5$ & -27 & $6.1 \pm 0.7$ & -11 & $3.7 \pm 0.4$ & 0 & $7.1 \pm 0.4$ & -25 \\
\hline $18: 1 \mathrm{n} 5$ & $0.4 \pm 0.1$ & -5 & $0.5 \pm 0.1$ & -2 & $0.5 \pm 0.0$ & 0 & $0.8 \pm 0.2$ & -17 & $0.7 \pm 0.1$ & -12 & $0.9 \pm 0.2$ & -23 & $0.6 \pm 0.2$ & -6 & $0.4 \pm 0.1$ & -3 & $0.3 \pm 0.0$ & -9 \\
\hline $18: 2 \mathrm{n} 6^{\mathrm{b}}$ & $0.9 \pm 0.2$ & -18 & $1.1 \pm 0.2$ & -11 & $0.6 \pm 0.1$ & -23 & $0.7 \pm 0.2$ & -24 & $1.2 \pm 0.1$ & -5 & $1.1 \pm 0.1$ & -12 & $1.0 \pm 0.2$ & -8 & $0.5 \pm 0.1$ & -33 & $1.1 \pm 0.1$ & -10 \\
\hline $18: 3 n 3$ & $0.3 \pm 0.1$ & -8 & $0.5 \pm 0.1$ & -2 & $0.2 \pm 0.0$ & -12 & $0.2 \pm 0.1$ & -12 & $0.3 \pm 0.0$ & -6 & $0.4 \pm 0.1$ & -5 & $0.3 \pm 0.1$ & -6 & $0.1 \pm 0.0$ & -21 & $0.4 \pm 0.0$ & -3 \\
\hline $18: 4 \mathrm{n} 3^{\mathrm{b}}$ & $1.0 \pm 0.3$ & -6 & $1.3 \pm 0.5$ & -3 & $0.4 \pm 0.1$ & -10 & $0.4 \pm 0.1$ & -13 & $0.9 \pm 0.2$ & -5 & $0.7 \pm 0.2$ & -10 & $0.6 \pm 0.2$ & -7 & $0.1 \pm 0.0$ & -18 & $1.8 \pm 0.2$ & 0 \\
\hline $20: 0^{\mathrm{a}}$ & $0.1 \pm 0.0$ & -7 & $0.0 \pm 0.0$ & -12 & $0.1 \pm 0.0$ & 0 & $0.1 \pm 0.0$ & -2 & $0.0 \pm 0.0$ & -12 & $0.1 \pm 0.0$ & 0 & $0.1 \pm 0.0$ & -2 & $0.1 \pm 0.0$ & -4 & $0.1 \pm 0.0$ & -8 \\
\hline $20: 1 \mathrm{n} 11^{\mathrm{b}}$ & $0.7 \pm 0.2$ & -16 & $0.9 \pm 0.3$ & -13 & $0.6 \pm 0.2$ & -13 & $1.4 \pm 0.3$ & -4 & $1.4 \pm 0.4$ & -3 & $1.1 \pm 0.2$ & -10 & $1.0 \pm 0.2$ & -8 & $0.4 \pm 0.1$ & -22 & $0.3 \pm 0.2$ & -24 \\
\hline $20: 1 \mathrm{n} 9^{\mathrm{b}}$ & $5.4 \pm 2.2$ & -15 & $11.4 \pm 4.2$ & -2 & $2.2 \pm 0.7$ & -17 & $1.3 \pm 0.2$ & -24 & $4.1 \pm 1.1$ & -14 & \pm 2.1 & -11 & \pm 2.0 & -10 & $.4 \pm 0.7$ & -23 & \pm 0.9 & -15 \\
\hline $20: 1 \mathrm{n} 7^{\mathrm{b}}$ & $0.6 \pm 0.4$ & -1 & $0.3 \pm 0.1$ & -7 & $2.6 \pm 1.0$ & -31 & $2.9 \pm 0.4$ & -62 & $1.4 \pm 0.1$ & -40 & $1.1 \pm 0.1$ & -35 & $1.2 \pm 0.6$ & -16 & $1.7 \pm 0.8$ & -21 & $0.5 \pm 0.1$ & -1 \\
\hline $20: 2 \mathrm{n} 6$ & $0.3 \pm 0.1$ & 0 & $0.3 \pm 0.1$ & -1 & $0.2 \pm 0.0$ & -9 & $0.5 \pm 0.1$ & -19 & $0.3 \pm 0.1$ & -1 & $0.3 \pm 0.1$ & -6 & $0.4 \pm 0.2$ & -8 & $0.2 \pm 0.1$ & -14 & $0.4 \pm 0.1$ & -6 \\
\hline $20: 4 \mathrm{n} 6$ & $1.2 \pm 0.2$ & -45 & $0.7 \pm 0.3$ & -16 & $4.0 \pm 1.1$ & -47 & $4.0 \pm 0.8$ & -58 & $2.5 \pm 0.4$ & -58 & $2.5 \pm 0.7$ & -41 & $3.2 \pm 1.0$ & -40 & $5.6 \pm 0.5$ & -88 & $0.5 \pm 0.0$ & -15 \\
\hline $20: 3 n 3^{a}$ & $0.1 \pm 0.0$ & -5 & $0.1 \pm 0.0$ & 0 & $0.1 \pm 0.0$ & -1 & $0.1 \pm 0.0$ & -16 & $0.1 \pm 0.0$ & -1 & $0.1 \pm 0.0$ & -2 & $0.2 \pm 0.1$ & -10 & $0.1 \pm 0.0$ & -3 & $0.2 \pm 0.0$ & -43 \\
\hline $20: 4 n 3^{b}$ & $0.4 \pm 0.1$ & -3 & $0.5 \pm 0.1$ & 0 & $0.2 \pm 0.1$ & -11 & $0.2 \pm 0.1$ & -14 & $0.6 \pm 0.1$ & 0 & $0.6 \pm 0.1$ & -2 & $0.3 \pm 0.1$ & -6 & $0.1 \pm 0.1$ & -18 & $0.5 \pm 0.0$ & 0 \\
\hline $20: 5 \mathrm{n} 3^{\mathrm{b}}$ & $12.4 \pm 1.5$ & -34 & $10.9 \pm 2.0$ & -26 & $16.6 \pm 2.0$ & -38 & $13.3 \pm 5.1$ & -22 & $14.9 \pm 0.9$ & -35 & $9.6 \pm 0.8$ & -24 & $10.0 \pm 1.7$ & -18 & $14.0 \pm 1.4$ & -40 & $9.3 \pm 0.2$ & -23 \\
\hline $22: 1 \mathrm{n} 11^{\mathrm{b}}$ & \pm 1.8 & -6 & \pm 2.9 & 0 & $=1.2$ & -7 & \pm 0.1 & -14 & 0.5 & -8 & 0.7 & -8 & 1.1 & -6 & \pm 0.2 & -14 & 0.5 & -6 \\
\hline $22: 1 \mathrm{n} 9^{\mathrm{b}}$ & $0.8 \pm 0.2$ & -2 & $1.1 \pm 0.3$ & -1 & $0.9 \pm 0.1$ & 0 & $0.5 \pm 0.1$ & -7 & $0.4 \pm 0.1$ & -6 & $0.9 \pm 0.1$ & 0 & $0.8 \pm 0.2$ & -1 & $0.3 \pm 0.0$ & -14 & $0.7 \pm 0.1$ & -2 \\
\hline $22: 1 \mathrm{n} 7^{\mathrm{a}}$ & $0.2 \pm 0.1$ & -6 & $0.3 \pm 0.1$ & -11 & $1.1 \pm 0.1$ & -64 & $0.8 \pm 0.3$ & -30 & $0.4 \pm 0.1$ & -18 & $0.2 \pm 0.0$ & -11 & $0.5 \pm 0.2$ & -24 & $1.3 \pm 0.4$ & -40 & $0.2 \pm 0.0$ & -3 \\
\hline $21: 5 n 3$ & $0.3 \pm 0.1$ & -4 & $0.3 \pm 0.0$ & -4 & $0.2 \pm 0.0$ & -5 & $0.3 \pm 0.1$ & -5 & $0.4 \pm 0.0$ & -1 & $0.2 \pm 0.0$ & -7 & $0.2 \pm 0.0$ & -5 & $0.2 \pm 0.1$ & -11 & $0.3 \pm 0.0$ & -2 \\
\hline $22: 4 \mathrm{n} 6$ & $0.3 \pm 0.1$ & -3 & $0.1 \pm 0.0$ & -6 & $0.6 \pm 0.2$ & -18 & $1.1 \pm 0.5$ & -26 & $0.3 \pm 0.0$ & -2 & $0.2 \pm 0.0$ & 0 & $0.5 \pm 0.2$ & -10 & $0.5 \pm 0.1$ & -18 & $0.2 \pm 0.0$ & -2 \\
\hline $22: 5 \mathrm{n} 6^{\prime}$ & $0.4 \pm 0.1$ & -22 & $0.1 \pm 0.1$ & 0 & $0.4 \pm 0.1$ & -22 & $0.3 \pm 0.1$ & -20 & $0.2 \pm 0.0$ & -6 & $0.2 \pm 0.0$ & -17 & $0.3 \pm 0.1$ & -14 & $0.4 \pm 0.1$ & -31 & $0.1 \pm 0.0$ & -3 \\
\hline $22: 5 n 3$ & $1.0 \pm 0.1$ & -16 & $1.0 \pm 0.3$ & -16 & $2.9 \pm 0.7$ & -4 & $2.7 \pm 1.0$ & -6 & $2.2 \pm 0.1$ & -6 & $1.2 \pm 0.1$ & -15 & $1.2 \pm 0.3$ & -10 & $2.7 \pm 0.4$ & -7 & $0.9 \pm 0.0$ & -17 \\
\hline $24: 0^{\mathrm{a}}$ & $0.1 \pm 0.0$ & -17 & $0.1 \pm 0.0$ & -4 & $0.2 \pm 0.0$ & -25 & $0.1 \pm 0.0$ & -24 & $0.1 \pm 0.1$ & -6 & $0.1 \pm 0.0$ & -6 & $0.1 \pm 0.0$ & -12 & $0.2 \pm 0.1$ & -24 & $0.0 \pm 0.0$ & 0 \\
\hline $22: 6 \mathrm{n} 3^{\mathrm{b}}$ & $19.2 \pm 3.4$ & -26 & $14.4 \pm 4.0$ & -9 & $14.8 \pm 3.0$ & -10 & $11.2 \pm 2.2$ & -1 & $11.5 \pm 0.9$ & -2 & $11.5 \pm 1.5$ & -2 & $12.2 \pm 0.9$ & -4 & $20.9 \pm 1.4$ & -38 & $8.9 \pm 0.7$ & -2 \\
\hline $24: 1 \mathrm{n} 9$ & $1.7 \pm 0.4$ & -32 & $1.3 \pm 0.5$ & -21 & $2.2 \pm 0.9$ & -27 & $1.3 \pm 0.3$ & -25 & $1.0 \pm 0.1$ & -15 & $1.2 \pm 0.4$ & -20 & $2.0 \pm 0.4$ & -34 & $3.1 \pm 0.7$ & -47 & $0.8 \pm 0.1$ & -12 \\
\hline$\Sigma$ SAFA & 23.8 & & 21.2 & & 22.9 & & 25.0 & & 21. & & 21. & & 22.7 & & 26.9 & & 28.4 & \\
\hline$\Sigma$ MUFA & 37.0 & & 46.2 & & 34.4 & & 38.2 & & 42.5 & & 48.6 & & 45.4 & & 26.4 & & 44.9 & \\
\hline$\Sigma$ PUFA & 38.4 & & 32.2 & & 42.5 & & 36.5 & & 36.2 & & 29.4 & & 31.4 & & 46.7 & & 25.3 & \\
\hline$\%$ total FA & $1.50 \pm 0.5$ & & $3.10 \pm 0.9$ & & $1.30 \pm 0.3$ & & $1.3 \pm 0.2$ & & $2.2 \pm 0.8$ & & $2.4 \pm 0.8$ & & $1.1 \pm 0.1$ & & $0.7 \pm 0.3$ & & $3.0 \pm 0.5$ & \\
\hline$\%$ cholestero & ol $0.19 \pm 0.0$ & & $0.90 \pm 0.0$ & & $0.60 \pm 0.0$ & & $0.2 \pm 0.0$ & & $0.2 \pm 0.1$ & & $0.2 \pm 0.0$ & & $0.2 \pm 0.0$ & & $0.1 \pm 0.0$ & & $0.1 \pm 0.0$ & \\
\hline
\end{tabular}




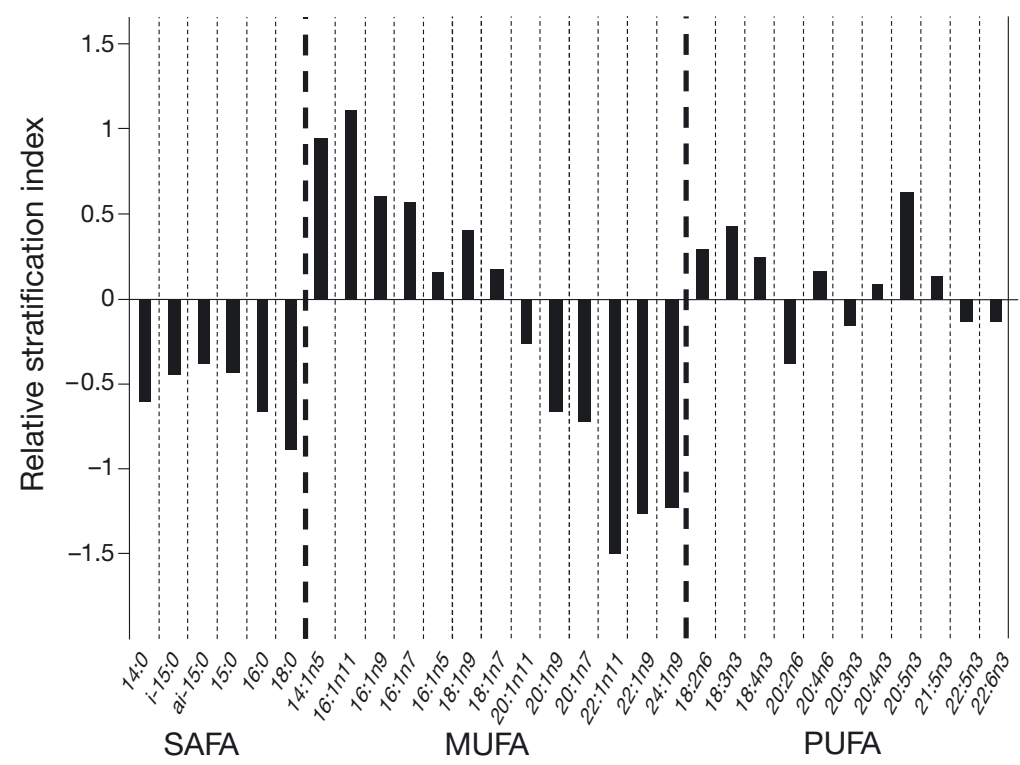

Fig. 2. Pagophilus groenlandicus. Stratification index for 30 fatty acids (FAs) in harp seal blubber obtained by subtracting the percentage in inner blubber layer from the percentage in the outer blubber layer (Table 1) and dividing the difference by the mean of the percentages in the inner and outer layers. MUFA: monounsaturated FA; PUFA: polyunsaturated FA; SAFA: saturated FA

hauls fell (Fig. 7). The pelagic amphipod samples, which overlap the inner blubber in the PC plot in Fig. 6, were more remote from the inner blubber than the outer blubber samples. The distance between the inner blubber and the prey species increased gradually from pelagic amphipods to long rough dab, but at larger increments among the last 6 to 7 species (Fig. 7).

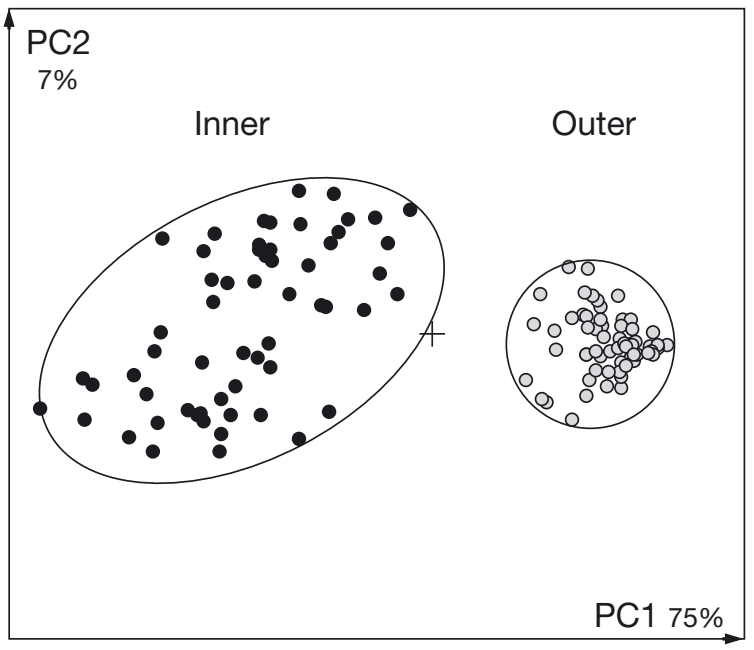

Fig. 3. Pagophilus groenlandicus. Principal components (PCs) plot of blubber samples from 57 harp seals. The percentage of the total variation among the samples along each PC is given. The origin of the plot is marked with a cross

\section{DISCUSSION}

The stratification of the blubber, with systematically higher levels of the shortchained MUFAs with 14 to 18 carbons in the outer layer (Fig. 2), was similar to the stratification found in blubbers of other aquatic mammals, e.g. harbour porpoise Phocoena phocoena (Koopman et al. 1996), dusky dolphin Lagenorhynchus obscures (Grahl-Nielsen et al. 2010), Baikal seal Phoca sibirica (Grahl-Nielsen et al. 2005), harbour seal Phoca vitulina (Andersen et al. 2004), Atlantic walrus Odobenus rosmarus rosmarus (Skoglund et al. 2010), southern elephant seal Mirounga leonine (Best et al. 2003), minke whale Balaenoptera acutorostrata (Olsen \& Grahl-Nielsen 2003) and ringed seal Pusa hispida (Strandberg et al. 2008). It has been suggested that the FA composition of the outer blubber layer is phylogenetically determined to serve functional roles related to insulation and streamlining (Lockyer et al. 1984, Aguilar \& Borrell 1990, Best et al. 2003, Struntz et al. 2004, Koopman 2007, Strandberg et al. 2008). The lower variability of the FA compositions of the outer layer relative to the inner layer supported the idea that the outer layer serves a functional role. The

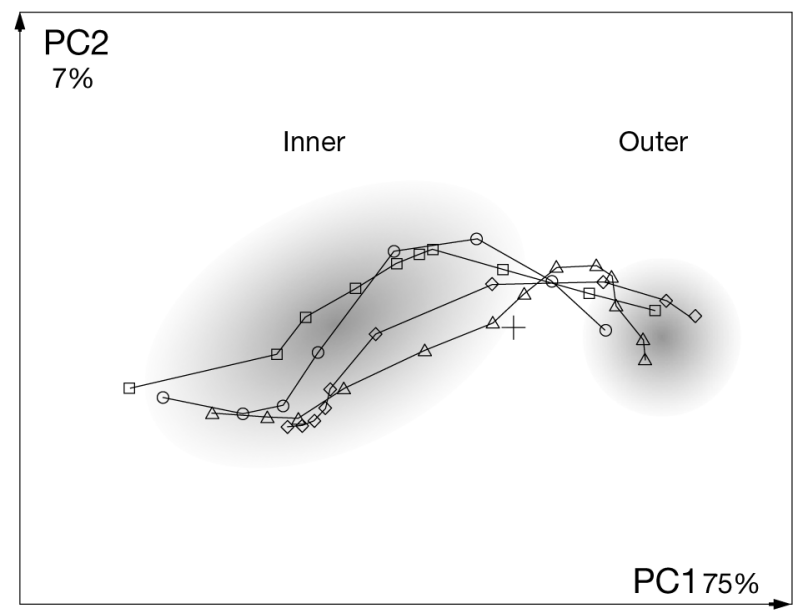

Fig. 4. Pagophilus groenlandicus. Principal components (PC) plot of sections of even thickness of $2 \mathrm{~mm}$ through the blubber column of 4 harp seals Pagophilus groenlandicus, from the innermost on the left to the outermost on the right. Due to differences in thickness of the blubber in the 4 animals, the number of subsamples ranges from 8 (circles) to 13 (triangles). These stratification samples were projected onto the PC plot of the inner and outer blubber samples in Fig. 2 without having any influence on the computation of the PCs. The inner and outer blubber samples are located within the shaded areas. The origin of the plot is marked with a cross 


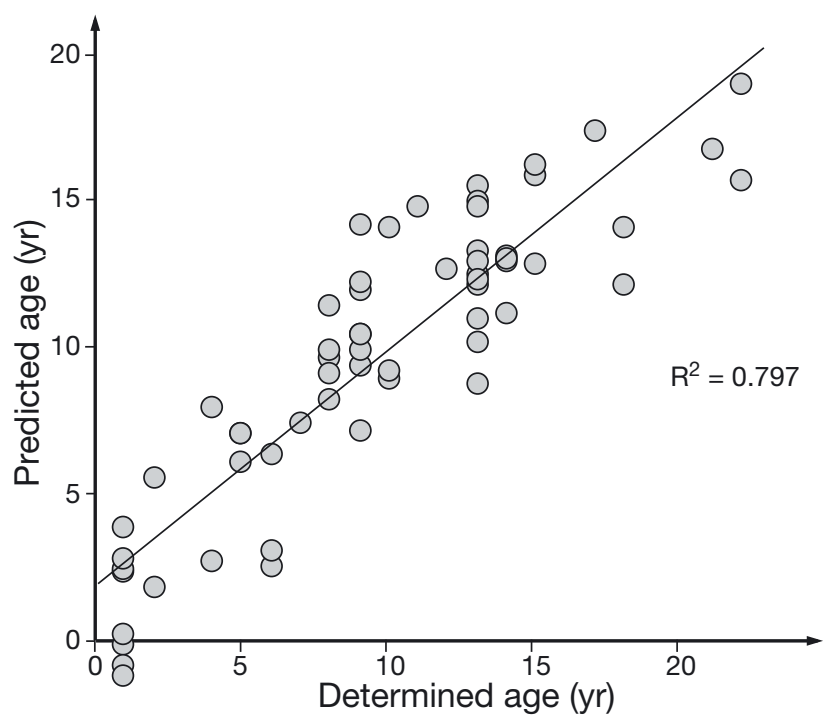

Fig. 5. Pagophilus groenlandicus. Predicted versus determined harp seal age (yr) based on partial least square regression of the fatty acid composition in the outer blubber layer inborne control of the outer blubber layer, leading to similar FA compositions in species with large differences in foraging ecology, indicates an absence of impact of the diet on the FA composition.

In contrast, the results in Fig. 5 show that there was a positive age effect on the FA composition of the outer blubber layer. Koopman et al. (1996) found a correlation between FA levels in the outer blubber layer of male harbour porpoises and age, particularly for isovaleric acid $\left(R^{2}=0.764\right)$. Strandberg et al. (2008) demonstrated a correlation between age $\left(\mathrm{R}^{2}=0.87\right)$ and the $\Delta 9$-desaturation index of the outermost layer of ventral blubber of ringed seals. Further, Herman et al. (2008) showed a correlation between age $\left(\mathrm{R}^{2}=0.89\right)$ and 15 and 16 carbon straight-chain and branched SAFAs in the outer layer of killer whales Orcinus orca, and Skoglund at al. (2010) described a correlation between age $\left(R^{2}=0.893\right)$ and the FA composition of the outer blubber layer of Atlantic walrus. This systematic change with age of the FA composition in the outer blubber layer substantiates the inference made above

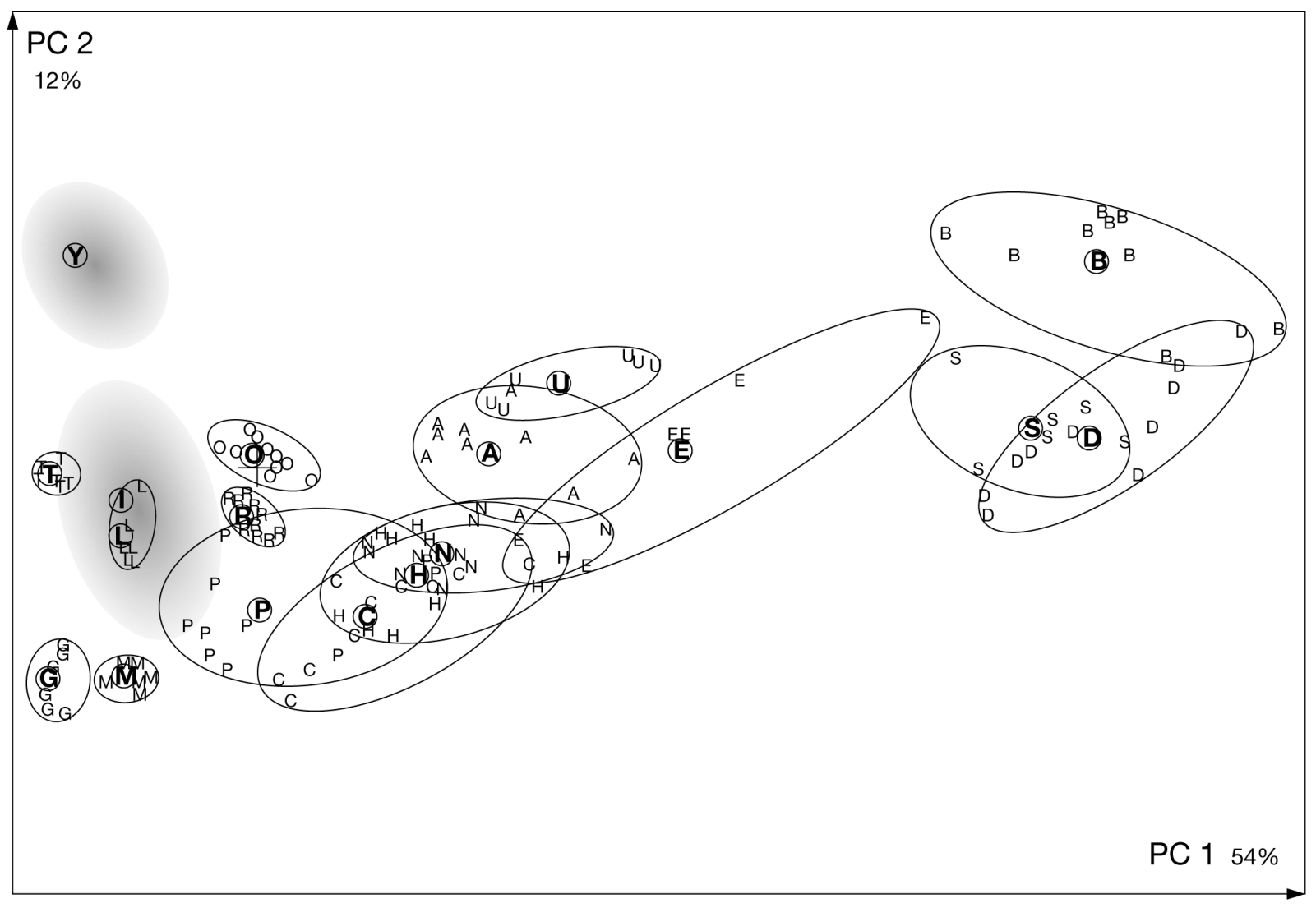

Fig. 6. Pagophilus groenlandicus. Principal components (PCs) plot of samples of inner and outer blubber of harp seals and of 16 prey species. Each letter represents one sample. The mean values from Table 1, encircled and in bold, are projected onto the plot without having an influence on the PCs. The 57 samples from inner (I) and outer (Y) blubber, not shown individually, fall inside the shaded areas. The origin of the plot is marked with a cross. A: Atlantic poacher; B: snake blenny; C: capelin; D: long rough dab; E: eelpout; G: Gammarus wikitzkii; H: haddock; L: Parathemisto libellula; M: Meganyctiphanes norvegica; N: Norway pout; O: Arctid cod; P: polar cod; R: Greenland halibut; S: spotted snake blenny; T: Thysanoessa inermis; U: Atlantic hooker sculpin 


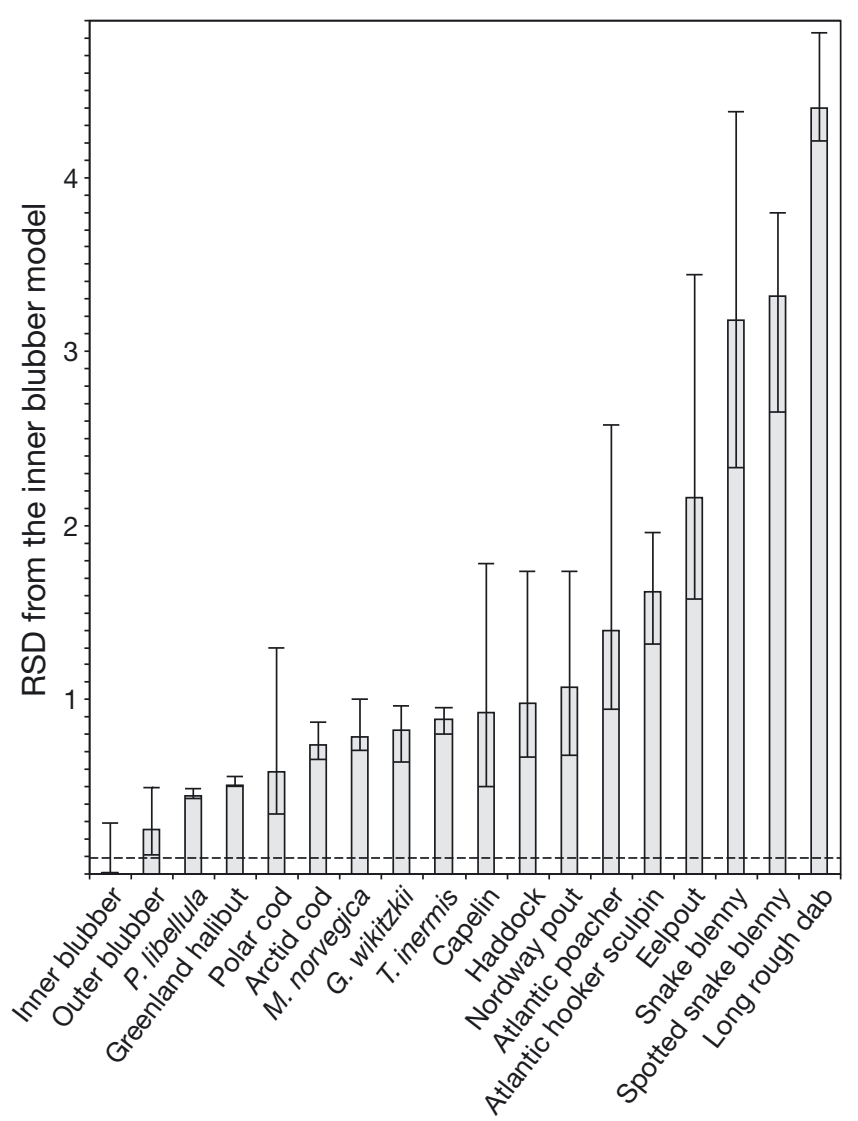

Fig. 7. Pagophilus groenlandicus. Relative standard deviation (RSD) of the mean values of the harp seal blubber and prey samples (Table 1, see also genus abbreviations there) from a model of the inner blubber samples based on 34 fatty acids. The model has 6 significant components (as tested by cross validation), covering $87 \%$ of the total variance among the samples. The outer limit of the model (95\% confidence limit) is at RSD $=0.09$, shown by a dashed line. Error bars indicate the lowest and highest RSD of individual samples

that this layer is independent of changes in the quality and quantity of prey.

The inner blubber layer is the most metabolically active part of the blubber, and this layer should be used to search for possible links to the diet. It is therefore not appropriate to sample the whole blubber column when the purpose of a study is to find information about the diet, because possible information about the diet that might lie in the FAs in the inner layer, which in that case will be masked by 'non-dietary' FAs from the outer layer. Smith \& Worthy (2006) also emphasised that it is important that the innermost layer of the blubber is analysed when FA data are used to make dietary inferences.

Certainly, harp seals have distinct seasonal variation in their blubber thickness and body condition, with maximum thickness in the autumn and winter and minimum in late spring (Nilssen et al. 1997). This reflects the dynamics of the lipid transfer in the Arctic marine ecosystem, where the energy produced during the algal bloom is transferred as FAs up through the food chain during summer and autumn to top predators, such as seals (e.g. Falk-Petersen et al. 1990, 2009). The harp seals used in the present study were sampled in May-June, i.e. at the beginning of their intensive summer feeding period, but before the beginning of their energy storage in form of blubber. We assume, however, that the active inner parts of the blubber core are most likely to be influenced by the lipids coming from the most recent food consumption. This is supported by previous feeding experiments on harp seals, where it has been noted that changes in FA composition in blubber, reflecting dietary changes, occurred despite the fact that there was no deposition of fat, in fact, all animals lost fat (Kirsch et al. 2000). Apparently, some turnover and net deposition of FAs take place regardless of whether the seal is fattening, and it has been suggested that the appearance of new dietary FAs is evident within 1 to $2 \mathrm{wk}$ of a switch in diet (Kirsch et al. 2000, Iverson et al. 2004).

The FA compositions of the potential prey species from the trawl hauls were all different from that of the inner blubber (Table 1, Fig. 6). The difference was most pronounced for cholesterol, with considerable levels in all potential prey species but absence in the blubber. This showed a metabolic selection during the deposition of the lipid components in the blubber.

There were also large differences among the potential prey species. The prey species with FA compositions most similar to those of the inner blubber were pelagic amphipods, small krill, greenland halibut, cod, large krill, sympagic amphipods and polar cod, followed by capelin (Fig. 6). In fact, pelagic amphipods were not distinct at all from the inner blubber in the PC plot in Fig. 6; however, this plot does not show the true picture of the differences between the FA composition of the potential prey species and the inner blubber because it is a projection of the samples onto the PC1-PC2 plane, which only covers $66 \%$ of the total variance among the samples, and also because all samples contribute to the plot.

For identification purposes, it is more appropriate to compare the potential prey species with the model of the inner blubber, based solely on the blubber samples with no influence from the potential prey samples. The 7 potential prey species with the least difference to the inner blubber were projected onto the PC1 - PC2 plane of the inner blubber model (Fig. 8). They were all different from the inner blubber. The true distance between the potential prey samples and the inner blubber was computed as the RSD of the prey samples to the model of the inner blubber samples (Fig. 7), showing that they all were significantly outside the model 


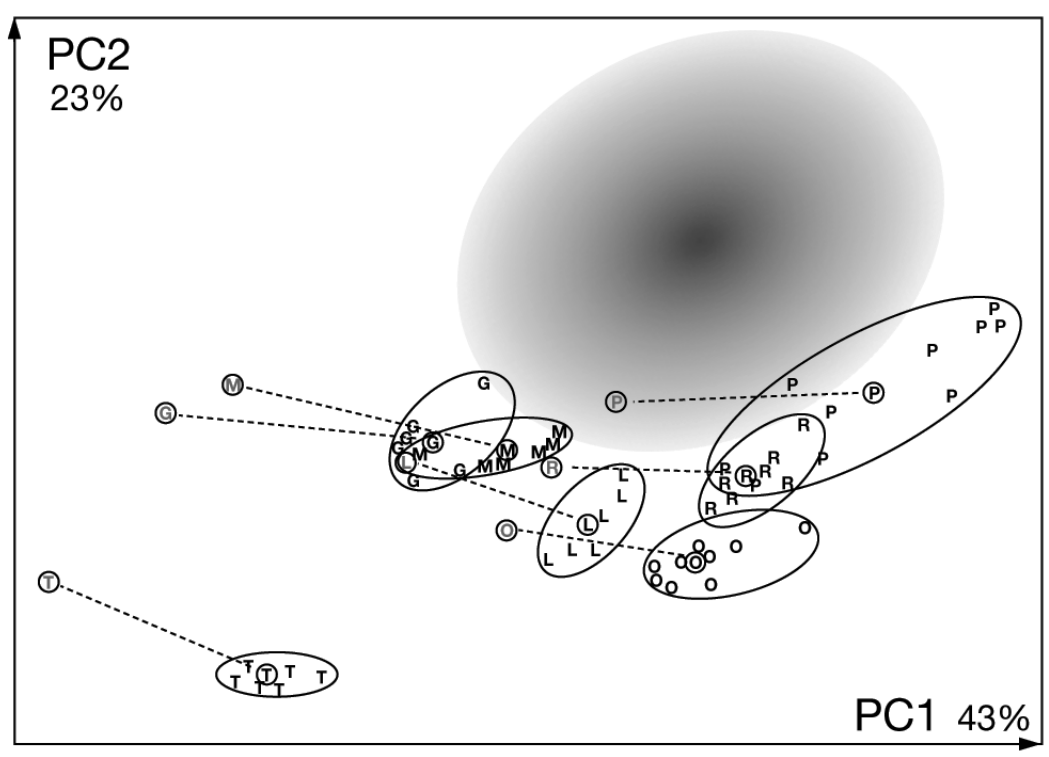

Fig. 8. Pagophilus groenlandicus. Principal components (PCs) plot of samples of the inner blubber of harp seals. All samples are located inside the shaded area. The samples from the 7 prey species closest to the inner blubber are projected onto the plot without having had any influence on the principal components. G: Gammarus wikitzkii; L: Parathemisto libellula; M: Meganyctiphanes norvegica; O: cod; P: polar cod; R: Greenland halibut; T: Thysanoessa inermis. Encircled letters indicate the means of the prey samples (Table 1) and the encircled grey letters indicate the mean prey samples after the fatty acid values were corrected with the harp seal calibration coefficients from Iverson et al. (2004)

ture analysis (QFASA) method, showing that harp seals from the western population consumed predominantly amphipods, polar cod, capelin and herring, in addition to sand lance Ammodytes spp. and redfish Sebastes spp. The hypothesis of the QFASA method is that if the predator is feeding on the same prey species over long enough time for complete exchange of the FAs in the blubber, i.e. some months, the FA composition of the blubber will attain the FA composition of the lipids in the prey, corrected by calibration coefficients. The coefficients are supposed to account for the effects of the predator's metabolism on the deposition of the FAs (Iverson et al. 2004, Tucker et al. 2009a,b). Feeding on several prey species will lead to a composition in the blubber that is an average of the inputs, corrected by the calibration coefficients, and weighted after the amount of lipid input from each prey.

Three sets of calibration coefficients have been established through feeding experiments on captive individuals (Iverson et al. 2004). Tucker et al.

( $p>0.05$ ). Still, investigations on stomach contents of harp seals from the Barents Sea (Lindstrøm et al. 2006, Pettersen 2007) have shown that their diet is comprised mainly of the prey species found here to have FA compositions closest to those of the inner blubber, i.e. polar cod, krill Parathemisto libellula and capelin.

With a clear distinction between the FA composition of the inner blubber and that of the potential prey species, it was obvious that the predator's metabolism had altered the different compositions from ingestion to deposition, resulting in a specific composition in the blubber. This is in accordance with the generally accepted view that the FA composition of blubber never matches that of the diet (Hooker et al. 2001, Best et al. 2003, Krahn et al. 2004, Iverson et al. 2004, Tucker et al. $2009 a, b)$. However, the resulting composition of the FAs in the inner blubber was not a weighted average of the potential prey FAs, because the prey species all were on the same side of the positions of the inner blubber samples in, although they were spread out. Thus, the composition of the inner blubber cannot be a product of the composition of the prey alone. There must also be an intrinsic factor affecting the metabolism towards a predetermined composition.

Some of these prey species appear to be present in the general diet of harp seals, as Tucker et al. (2009a) revealed by the use of the quantitative fatty acid signa- (2009a) have tried all 3 sets and combinations of them to discern the prey assemblage of harp seals from the western population. Although the results pointed to similar main prey species as have been found for the eastern population (Nilssen et al. 2000), the quantitative contribution from the various species was quite dispersed, i.e. amphipods were suggested to contribute between 0.1 and 0.5 parts of the total diet, Atlantic salmon Salmo salar between zero and 0.2 parts, capelin between zero and 0.1 parts and northern sand lance between zero and 0.2 parts. In addition, there were a large number of outliers, some of which indicated that the seals ate one species exclusively (Tucker et al. 2009a).

To investigate what calibration coefficients could accomplish with our data, we applied the harp seal calibration coefficients established by Iverson et al. (2004), which are based on captive juvenile harp seals fed with herring, to the FAs of the 7 potential prey species with FA compositions closest to that of the inner blubber. This resulted in a displacement of the prey species, with similar or increased distance to the inner blubber, but all still placed on the same side of the inner blubber model (Fig. 8). If the FA composition of the inner blubber should have resulted mainly from the prey, i.e. being a weighted average of the FA compositions of the prey, it should be expected that the 


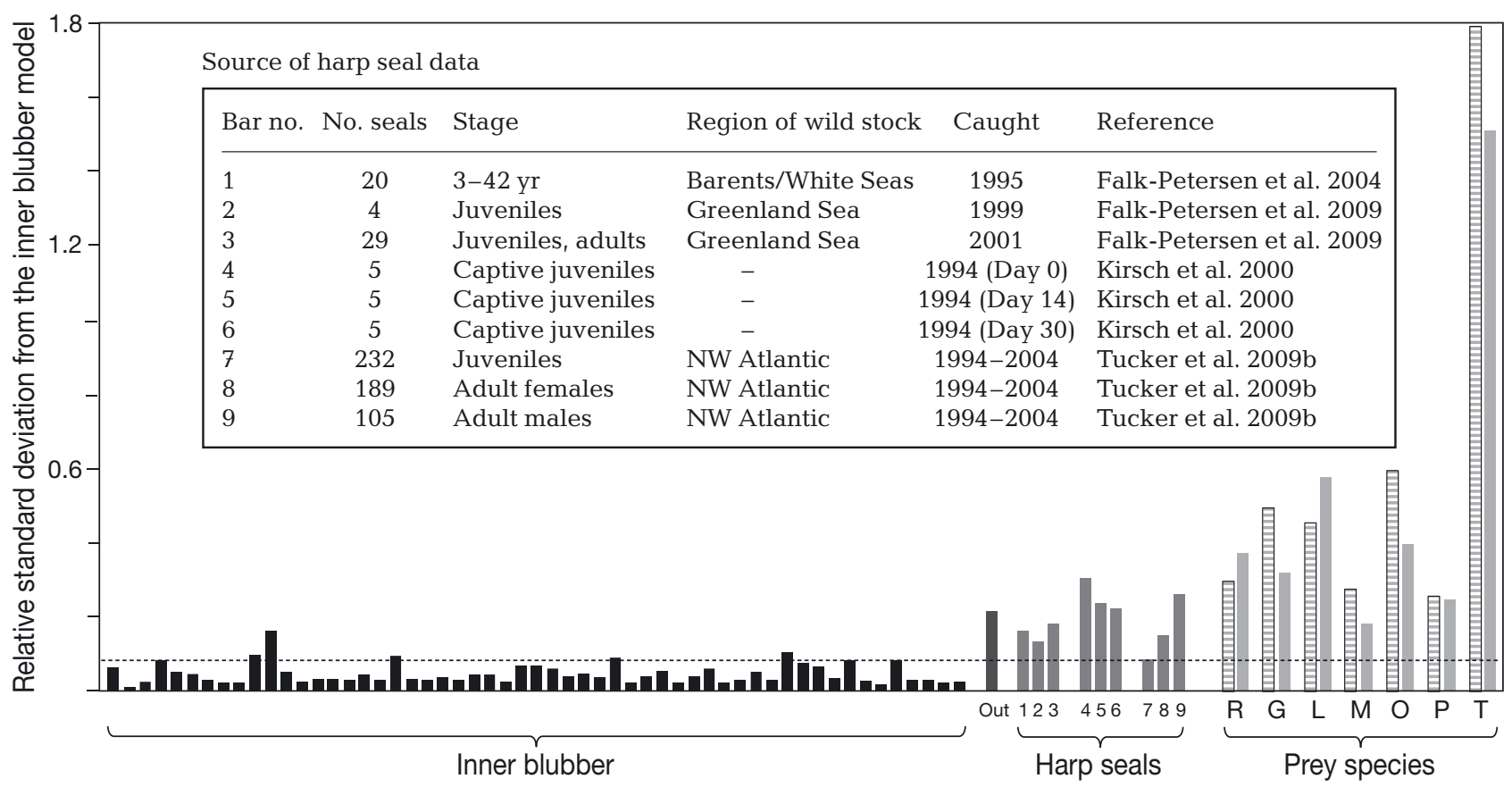

Fig. 9. Pagophilus groenlandicus. Relative standard deviation (RSD) of harp seal blubber and prey samples from the present study and of blubber samples from other investigations, as computed from a model of 55 inner blubber samples based on 10 fatty acids (FAs). The model has 6 significant components (as tested by cross validation), covering $97 \%$ of the total variance among the samples. The outer limit of the model is at RSD $=0.082$, shown by the dashed line. Each of the 55 inner blubber samples is shown whereas the mean of the 55 outer samples is presented (Out). Mean values of blubber samples from harp seals were determined from other investigations (inset). Prey species included are the 7 closest to the inner blubber in terms of FA composition. G: Gammarus wikitzkii; L: Parathemisto libellula; M: Meganyctiphanes norvegica; O: Arctic cod; P: polar cod; R: Greenland halibut; T: Thysanoessa inermis. Hatched bar: data from Table 1; filled light-grey bars: same data corrected with the harp seal calibration coefficients from Iverson et al. (2004)

prey samples should be located around the inner blubber model. With all prey species on the same side of the inner blubber it would not have been possible to quantify the contribution of these 7 prey species to the diet of the harp seals on the basis of the FA composition of the inner blubber, even if the relative fat content of each diet item was taken into consideration, as done by Iverson et al. (2004).

Still, the fact is that the most consumed prey species, as determined in the many investigations referred to above, are the ones which are most similar to the inner blubber with respect to FA composition (Figs. 6 \& 7). But this dietary effect must be inferior to the species effect on the final blubber FA composition.

The difference in FA composition between the inner blubber and the prey is caused by the different enzymes acting on the FAs through digestion, transport and deposition in the blubber. Thus, there is an intrinsic component responsible for the final FA composition of the blubber. It will always be possible to establish a coefficient for each FA that accounts for the prey-predator difference. But as the prey species have different compositions of their FAs, the coefficients will have different values for each species. Thus the same set of coefficients cannot be used for all prey species.
The final FA composition of the blubber is apparently dependent on the species of the predator. Four different reports on the FA composition of the blubber of harp seals have been published. Kirsch et al. (2000) studied 5 captive juvenile harp seals in 1994. Their results have given rise to calibration coefficients used in later studies (Iverson et al. 2004). Tucker et al. (2009b) have determined the FAs of blubber of 526 harp seals from the northwest Atlantic stock, captured between 1994 and 2004 along the coasts of Newfoundland, Labrador and Greenland. Falk-Petersen et al. (2004) analysed the blubber of 20 harp seals caught in the northern Barents Sea in 1995, and the same research group also studied 33 harp seals from the Greenland Sea stock, captured in 1999 and 2001 along the coast of Greenland from Fram Strait in the north to Denmark Strait, northwest of Iceland, in the south (Falk-Petersen et al. 2009).

We compared the results from these investigations with the present results by testing the mean values given in the 4 papers against the model of the inner blubber of the harp seals in the present investigation, sampled in the northwestern Barents Sea in 2006. Because there were differences in the selection of FAs between the investigations, 10 abundant FAs deter- 
mined in all investigations were used in this comparison. These FAs, marked in Table 1, were all dietary (Iverson et al. 2004). Although they were outside the outer limit for the blubber model ( $p=0.05)$, the distances (measured as RSDs) showed a remarkable similarity between harp seals from the 3 different stocks, sampled over a period of more than 10 yr (Fig. 9). Both spatial and temporal changes in consumed prey must have taken place, so this is a strong indication of a genetic effect on the FA composition of the seal blubber.

But even if the metabolism is the main determinant, an impact of the FAs from the prey cannot be excluded. Specific FAs, or ratios between specific FAs, might overrule the metabolism, according to the FA trophic marker concept (Dalsgaard et al. 2003). Thus, FalkPetersen et al. (2009) showed that the FAs in harp seal blubber originate from a diatom-dominated ecosystem due to the presence of 16:4n1. The rather high levels of the long-chained MUFAs 20:1 and 22:1 (Falk-Petersen et al. 2009) suggested a Calanus spp.-based ecosystem, as these FAs are formed de novo by Calanus spp. (Sargent \& Henderson 1986, Scott et al. 2002). The following prey species also had high levels of these FAs: large krill, sympagic amphipods, pelagic amphipods and polar cod. These species were among those which most resembled the inner blubber in terms of FA composition (Figs. $6 \&$ 7) and also among those previously found in the stomach contents of harp seals in the Barents Sea (Lindstrøm et al. 2006, Pettersen 2007).

The conclusion from the present study is that just as the prey species have distinct FA compositions, marine mammals have species-specific FA compositions of their blubber, with a minor impact of the diet. This is in support of our hypothesis. The metabolism will convert the dietary FA composition to a given blubber composition regardless of the FA composition of the prey. The outer blubber, in particular, is unique; it has specific physiological functions but a FA composition that changes with the age of the seals. The FA composition of the inner blubber, serving mainly as energy storage (receiving the lipids from the diet), is also determined by the metabolism, but still has a qualitative relationship with the FA composition of the prey. For example, particular trophic marker FAs might permeate this blubber layer. The relationship does, however, defy quantitative determination of the prey assemblage.

Acknowledgment. We thank S. Falk-Petersen for critically reviewing the manuscript.

\section{LITERATURE CITED}

Aguilar A, Borrell A (1990) Patterns of lipid content and stratification in the blubber of fin whales (Balaenoptera physalus). J Mammal 71:544-554
Andersen SM, Lydersen C, Grahl-Nielsen O, Kovacs KM (2004) Autumn diet of harbour seals (Phoca vitulina) at Prins Karls Forland, Svalbard, assessed via scat and fattyacid analyses. Can J Zool 82:1230-1245

Bergstad OA, Jørgensen T, Dragesund O (1987) Life history and ecology of the gadoid resources of the Barents Sea. Fish Res 5:119-161

Best NJ, Bradshaw CJA, Hindell MA, Nichols PD (2003) Vertical stratification of fatty acids in the blubber of southern elephant seals (Mirounga leonina): implications for diet analysis. Comp Biochem Physiol B 134:253-263

Birkeland A, Kovacs KM, Lydersen C, Grahl-Nielsen O (2005) Transfer of fatty acids from mothers to their calves during lactation in white whales Delphinapterus leucas. Mar Ecol Prog Ser 298:287-294

Bogstad B, Haug T, Mehl S (2000) Who eats whom in the Barents Sea? NAMMCO Sci Publ 2:65-80

> Bowen WD, Sergeant DE, Øritsland T (1983) Validation of age estimation in harp seals, Phoca groenlandica, using dental annuli. Can J Fish Aquat Sci 40:1430-1441

> Dalsgaard J, St. John M, Kattner G, Müller-Navarra D, Hagen W (2003) Fatty acid trophic markers in the pelagic marine environment. Adv Mar Biol 46:225-340

Falk-Petersen S, Hopkins CCE, Sargent JR (1990) Trophic relationships in the pelagic, Arctic food web. In: Barnes M, Gibson RN (eds) Trophic relationship in the marine environment. Aberdeen University Press, Aberdeen, p 315-333

Falk-Petersen S, Haug T, Nilssen KT, Wold A, Dahl TM (2004) Lipids and trophic linkages in harp seal (Phoca groenlandica) from the eastern Barents Sea. Polar Res 23:43-50

Falk-Petersen S, Haug T, Hop H, Nilssen KT, Wold A (2009) Transfer of lipids from plankton to blubber of harp and hooded seals off East Greenland. Deep-Sea Res II 56: 2080-2086

Folkow LP, Nordøy ES, Blix AS (2004) Distribution and diving behaviour of harp seals Pagophilus groenlandicus from the Greenland Sea stock. Polar Biol 27:281-298

Gjøsæter H (1998) The population biology and exploitation of capelin (Mallotus villosus) in the Barents Sea. Sarsia 83: 453-496

Gjøsæter H, Bogstad B, Tjelmeland S (2009) Ecosystem effects of the three capelin stock collapses in the Barents Sea. Mar Biol Res 5:40-53

> Grahl-Nielsen O, Mjaavatten O (1991) Dietry influence on fatty acid composition of blubber fat of seals as determined by biopsy: a multivariate approach. Mar Biol 110: $59-64$

Grahl-Nielsen O, Hammill MO, Lydersen C, Wahlstrøm S (2000) Transfer of fatty acids from female seal blubber via milk to pup blubber. J Comp Physiol B 170:277-283

$>$ Grahl-Nielsen O, Halvorsen AK, Bodoev N, Averina L and others (2005) Fatty acid composition of the blubber of the Baikal seal Phoca sibirica and its marine relative, the ringed seal Phoca hispida. Mar Ecol Prog Ser 305: 261-274

Grahl-Nielsen O, Krakstad JO, Nøttestad L, Axelsen BE (2010) Dusky dolphins (Lagenorhynchus obscurus) and Cape fur seals (Arctocephalus pusillus pusillus): fatty acid composition of their blubber and prey species. Afr J Mar Sci 32:187-196

> Haug T, Nilssen KT, Øien N, Potelov V (1994) Seasonal distribution of harp seals (Phoca groenlandica) in the Barents Sea. Polar Res 13:163-172

Haug T, Nilssen KT, Lindblom L (2004) Feeding habits of harp and hooded seals in drift ice waters along the east coast of Greenland in summer and winter. Polar Res 23:35-42 
Herman DP, Matkin CO, Ylitalo GM, Durban JW and others (2008) Assessing age distributions of killer whale Orcinus orca populations from the composition of endogeneous fatty acios in their outer blubber layers. Mar Ecol Prog Ser 372:289-302

> Hooker SK, Iverson SJ, Ostrom P, Smith SC (2001) Diet of northern bottlenose whales inferred from fatty-acid and stable-isotope analyses of biopsy samples. Can J Zool 79:1442-1454

Iverson SJ, Field C, Bowen WD, Blanchard W (2004) Quantitative fatty acid signature analysis: a new method of estimating predator diets. Ecol Monogr 74:211-235

Kirsch PE, Iverson SJ, Don Bowen W (2000) Effect of a low-fat diet on body composition and blubber fatty acids of captive juvenile harp seals (Phoca groenlandica). Physiol Zool 73:45-59

Koopman HN (2007) Phylogenetic, ecological and ontogenetic factors influencing the biochemical structure of the blubber of odontocetes. Mar Biol 151:277-291

Koopman HN, Iverson SJ, Gaskin DE (1996) Stratification and age-related differences in blubber fatty acids of the male harbour porpoise (Phocoena phocoena). J Comp Physiol B 165:628-639

Krahn MM, Herman DP, Ylitalo GM, Sloan CA and others (2004) Stratification of lipids, fatty acids and organochlorine contaminats in blubber of white whales and killer whales. J Cetacean Res Manag 6:175-189

Kvalheim OM, Karstang TV (1987) A general purpose program for multivariate data analysis. Chemometr Intell Lab Syst 2:235-237

Kwetegyeka J, Mpango G, Grahl-Nielsen O (2008) Variation in fatty acid composition in muscle and heart tissues among species and populations of tropical fish in Lakes Victoria and Kyoga. Lipids 43:1017-1029

Lavigne DM, Kovacs KM (1988) Harps and hoods. Ice-breeding seals of the northwest Atlantic. University of Waterloo Press, Waterloo, ON

Lindstrøm U, Harbitz A, Haug T, Nilssen KT (1998) Do harp seals Phoca groenlandica exhibit particular prey preferences? ICES J Mar Sci 55:941-953

Lindstrøm U, Nilssen KT, Haug T (2006) Prey consumption by harp seals in the Barents Sea in 1995-2005. ICES CM 2006/L:08. ICES, Copenhagen

- Lockyer C, McDonnell LC, Waters TD (1984) The biochemical composition of fin whale blubber. Can J Zool 62:2553-2562

- Meier S, Mjøs SA, Joensen H, Grahl-Nielsen O (2006) Validation of a one-step extraction/methylation method for determination of fatty acids and cholesterol in marine tissues. J Chromatogr A 1104:291-298

Nilssen KT, Haug T, Potelov V, Timoshenko YK (1995) Food habits and food availability of harp seals (Phoca groenlandica) during early summer and autumn in the northern Barents Sea. Polar Biol 15:485-493

Nilssen KT, Haug T, Grotnes PE, Potelov VA (1997) Seasonal variation in body condition of adult Barents Sea harp seals (Phoca groenlandica). J Northwest Atl Fish Sci 22:17-25

Nilssen KT, Pedersen OP, Folkow L, Haug T (2000) Food con-

Editorial responsibility: Michael Castellini,

Fairbanks, Alaska, USA sumption estimates of Barents Sea harp seals. NAMMCO Sci Publ 2:9-28

Nordøy ES, Folkow LP, Potelov V, Prischemikhin V, Blix AS (2008) Seasonal distribution and dive behaviour of harp seals (Pagophilus groenlandicus) of the White Sea-Barents Sea stock. Polar Biol 31:1119-1135

Nordstrom CA, Wilson LJ, Iverson SJ, Tollit DJ (2008) Evaluating quantitative fatty acid signature analysis (QFASA) using harbour seals Phoca vitulina richardsi in captive feeding studies. Mar Ecol Prog Ser 360:245-263

Olsen E, Grahl-Nielsen O (2003) Blubber fatty acids of minke whales: stratification, population identification and relation to diet. Mar Biol 142:13-24

Pettersen LMS (2007) Grønlandsselens (Pagophilus groenlandicus) beiting om sommeren i åpent farvann i svalbardområdet. MSc thesis, University of Tromsø

Potelov VA, Golikov AP, Bondarev VA (2003) Estimated pup production of harp seals Pagophilus groenlandicus in the White Sea, Russia, in 2000. ICES J Mar Sci 60:1012-1017

Sargent JR, Henderson RJ (1986) Lipids. In: Corner EDS, O'Hara SCM (eds) The biological chemistry of marine copepods. Clarendon Press, Oxford, p 59-164

> Scott CL, Kwasniewski S, Falk-Petersen S, Sargent JR (2002) Species differences, origins and functions of fatty alcohols and fatty acids in the wax esters and phospholipids of Calanus hyperboreus, C. glacialis and C. finmarchicus from Arctic waters. Mar Ecol Prog Ser 235:127-134

Sergeant DE (1991) Harp seal, man and ice. Can Fish Aquat Sci Spec Publ 114:1-153

Skoglund EG, Lydersen C, Grahl-Nielsen O, Haug T, Kovacs KM (2010) Fatty acid composition of the blubber and dermis of the Atlantic walrus (Odobenus rosmarus rosmarus) in Svalbard, and its potential prey. Mar Biol Res 6:239-250

Smith HR, Worthy GAJ (2006) Stratification and intra- and inter-specific differences in fatty acid composition of common dolphin (Delphinus sp.) blubber: implications for dietary analysis. Comp Biochem Physiol B 143:486-499

Strandberg U, Käkelä A, Lydersen C, Kovacs KM, GrahlNielsen O, Hyvärinen H, Käkelä R (2008) Stratification, composition and function of marine mammal blubber: the ecology of fatty acids in marine mammals. Physiol Biochem Zool 81:473-485

> Struntz DJ, McLellan WA, Dillaman RM, Blum JE, Kucklick JR, Pabst DA (2004) Blubber development in bottlenose dolphins (Tursiops truncatus). J Morphol 259:7-20

Tollit DJ, Wong M, Winship AJ, Rosen DAS, Trites AWE (2003) Quantifying errors associated with using prey skeletal structures from fecal samples to determine the diet of Stellar's sea lion (Eumetropias jubatus). Mar Mamm Sci 19:724-744

Tucker S, Bowen WD, Iverson SJ, Blanchard W, Stenson GB (2009a) Sources of variation in diets of harp and hooded seals estimated from quantitative fatty acid signature analysis (QFASA). Mar Ecol Prog Ser 384:287-302

Tucker S, Bowen WD, Iverson SJ, Stenson GB (2009b) Intrinsic and extrinsic sources of variation in the diets of harp and hooded seals revealed by fatty acid profiles. Can J Zool 87:139-151

Submitted: August 25, 2010; Accepted: December 21, 2010 Proofs received from author(s): March 15, 2011 